\title{
Gabor windows supported on $[1,1]$ and construction of compactly supported dual windows with optimal smoothness
}

\author{
Lemvig, Jakob; Nielsen, Kamilla Haahr
}

Published in:

Journal of Approximation Theory

Link to article, DOI:

10.1016/j.jat.2019.105304

Publication date:

2020

Document Version

Peer reviewed version

Link back to DTU Orbit

Citation (APA):

Lemvig, J., \& Nielsen, K. H. (2020). Gabor windows supported on $[1,1]$ and construction of compactly supported dual windows with optimal smoothness. Journal of Approximation Theory, 249, [105304].

https://doi.org/10.1016/j.jat.2019.105304

\section{General rights}

Copyright and moral rights for the publications made accessible in the public portal are retained by the authors and/or other copyright owners and it is a condition of accessing publications that users recognise and abide by the legal requirements associated with these rights.

- Users may download and print one copy of any publication from the public portal for the purpose of private study or research.

- You may not further distribute the material or use it for any profit-making activity or commercial gain

- You may freely distribute the URL identifying the publication in the public portal

If you believe that this document breaches copyright please contact us providing details, and we will remove access to the work immediately and investigate your claim. 


\title{
Gabor windows supported on $[-1,1]$ and construction of compactly supported dual windows with optimal smoothness
}

\author{
Jakob Lemvig* \\ Technical University of Denmark, Department of Applied Mathematics and Computer Science, \\ 2800 Kgs. Lyngby, Denmark \\ Kamilla Haahr Nielsen \\ Technical University of Denmark, Department of Applied Mathematics and Computer Science, \\ 2800 Kgs. Lyngby, Denmark
}

\begin{abstract}
We consider Gabor frames $\left\{e^{2 \pi i b m} \cdot g(\cdot-a k)\right\}_{m, k \in \mathbb{Z}}$ with translation parameter $a=L / 2$, modulation parameter $b \in(0,2 / L)$ and a window function $g \in C^{n}(\mathbb{R})$ supported on $\left[x_{0}, x_{0}+L\right]$ and non-zero on $\left(x_{0}, x_{0}+L\right)$ for $L>0$ and $x_{0} \in \mathbb{R}$. The set of all dual windows $h \in L^{2}(\mathbb{R})$ with sufficiently small support is parametrized by 1-periodic measurable functions $z$. Each dual window $h$ is given explicitly in terms of the function $z$ in such a way that desirable properties (e.g., symmetry, boundedness and smoothness) of $h$ are directly linked to $z$. We derive easily verifiable conditions on the function $z$ that guarantee, in fact, characterize, compactly supported dual windows $h$ with the same smoothness, i.e., $h \in C^{n}(\mathbb{R})$. The construction of dual windows is valid for all values of the smoothness index $n \in \mathbb{Z}_{>0} \cup\{\infty\}$ and for all values of the modulation parameter $b<2 / L$; since $a=L / 2$, this allows for arbitrarily small redundancy $(a b)^{-1}>1$. We show that the smoothness of $h$ is optimal, i.e., if $g \notin C^{n+1}(\mathbb{R})$ then, in general, a dual window $h$ in $C^{n+1}(\mathbb{R})$ does not exist.
\end{abstract}

Keywords: dual frame, dual window, Gabor frame, optimal smoothness, redundancy 2010 MSC: Primary 42C15; Secondary 42A60

\section{Introduction}

One of the central tasks in signal processing and time-frequency analysis is to find convenient series expansions of functions in $L^{2}(\mathbb{R})$. A popular choice of such series expansions is by use of Gabor frames, which are function systems of the form

$$
\left\{M_{b m} T_{a k} g\right\}_{m, k \in \mathbb{Z}}=\left\{e^{2 \pi i b m \cdot} g(\cdot-a k)\right\}_{m, k \in \mathbb{Z}},
$$

\footnotetext{
${ }^{*}$ Corresponding author

Email addresses: jakle@dtu.dk (Jakob Lemvig), kamni@dtu.dk (Kamilla Haahr Nielsen)
} 
where $a, b>0$ and $g \in L^{2}(\mathbb{R})$, and where $T_{\lambda} f=f(\cdot-\lambda)$ and $M_{\gamma} f=e^{2 \pi i \gamma} f, \lambda, \gamma \in \mathbb{R}$, denote the translation and modulation operators on $L^{2}(\mathbb{R})$, respectively. Now, a Gabor frame for $L^{2}(\mathbb{R})$ is a Gabor system $\left\{M_{b m} T_{a k} g\right\}_{m, k \in \mathbb{Z}}$ for which there exist constants $A, B>0$ such that

$$
A\|f\|^{2} \leq \sum_{m, k \in \mathbb{Z}}\left|\left\langle f, M_{b m} T_{a k} g\right\rangle\right|^{2} \leq B\|f\|^{2} \quad \text { for all } \quad f \in L^{2}(\mathbb{R}) .
$$

If the upper bound holds, we say that $\left\{M_{b m} T_{a k} g\right\}_{m, k \in \mathbb{Z}}$ is a Bessel system with bound $B$. In case $a b<1$ and $\left\{M_{b m} T_{a k} g\right\}_{m, k \in \mathbb{Z}}$ satisfies (1.1), there exists infinitely many functions $h \in L^{2}(\mathbb{R})$ such that $\left\{M_{b m} T_{a k} h\right\}_{m, k \in \mathbb{Z}}$ is a Bessel system and

$$
f=\sum_{m, k \in \mathbb{Z}}\left\langle f, M_{b m} T_{a k} g\right\rangle M_{b m} T_{a k} h \text { for all } f \in L^{2}(\mathbb{R})
$$

holds with unconditionally $L^{2}$-convergence. The function $g$ generating the Gabor frame $\left\{M_{b m} T_{a k} g\right\}_{m, k \in \mathbb{Z}}$ is called the window, while $h$ is called a dual window. For a Bessel system $\left\{M_{b m} T_{a k} g\right\}_{m, k \in \mathbb{Z}}$, the linear operator $S_{g}: L^{2}(\mathbb{R}) \rightarrow L^{2}(\mathbb{R})$ defined by

$$
S_{g} f=\sum_{m, k \in \mathbb{Z}}\left\langle f, M_{b m} T_{a k} g\right\rangle M_{b m} T_{a k} g
$$

is called the frame operator, and the canonical dual window is given by $\tilde{h}=S_{g}^{-1} g$. We will consider windows $g$ supported on an interval of length $L$.

\subsection{Painless non-orthonormal expansions}

The most classical method of constructing dual windows is by painless non-orthonormal expansions by Daubechies et al. [11, Theorem 2]. Assume $s(x)$ is nonnegative, bounded and supported on an interval of length $L>0$, and that $s$ has constant periodization $\sum_{n \in \mathbb{Z}} s(x+a n)=1$ almost everywhere. Then defining $g=s^{p}$ and $h=s^{1-p}$, where $0<p<1$ classically is taken to be $p=1 / 2$, generate dual frames $\left\{M_{b m} T_{a k} g\right\}_{m, k \in \mathbb{Z}}$ and $\left\{M_{b m} T_{a k} h\right\}_{m, k \in \mathbb{Z}}$ for any $0<b \leq 1 / L$ and $0<a \leq L$.

A variant of the painless construction without assuming a partition of unity property (i.e., constant periodization) is as follows. We will again consider $g \in L^{\infty}(\mathbb{R})$ having compact support in an interval of length $L$. If $a \leq L$, and $b \leq 1 / L$, the frame operator $S_{g}$ becomes a multiplication operator:

$$
S_{g} f(x)=\frac{1}{b} \sum_{n \in \mathbb{Z}}|g(x+a n)|^{2} \cdot f(x) .
$$

It follows that the Gabor system $\left\{M_{b m} T_{a k} g\right\}_{m, k \in \mathbb{Z}}$ is a frame with bound $A$ and $B$ if and only if $A \leq \frac{1}{b} \sum_{n \in \mathbb{Z}}|g(\cdot+a n)|^{2} \leq B$, in which case the canonical dual window $\tilde{h}:=S_{g}^{-1} g$ is compactly supported on $\operatorname{supp} g$ and given by $\tilde{h}(x)=b g(x) / \sum_{n \in \mathbb{Z}}|g(x+a n)|^{2}$. In this case $\left\{M_{b m} T_{a k} g\right\}_{m, k \in \mathbb{Z}}$ and $\left\{M_{b m} T_{a k} \tilde{h}\right\}_{m, k \in \mathbb{Z}}$ are canonical dual Gabor frames with compact support in an interval of length $L$. 


\subsection{Our contribution}

On the borderline $a=L$ and $0<b<1 / L$ of the painless expansions region $(a, b) \in$ $(0, L] \times(0,1 / L]$, the discontinuous window $g=L^{-1 / 2} \mathbf{1}_{[0, L]}$ generates a tight Gabor frame. In this case, the Gabor system becomes a union of Fourier series $\bigcup_{k \in \mathbb{Z}}\left\{M_{b m} T_{a k} g\right\}_{m \in \mathbb{Z}}$ with no support overlap between the different Fourier systems $\left\{M_{b m} T_{a k} g\right\}_{m \in \mathbb{Z}}$ indexed by $k \in \mathbb{Z}$. This may lead to unwanted mismatch artifacts at the seam points $a \mathbb{Z}$ when truncating the Gabor expansion (1.2).

To diminish such artifacts, we will use a 2-overlap (or 2-covering) condition, namely, $a=L / 2$. This means that for almost any time $x \in \mathbb{R}$, two Fourier-like systems $\left\{M_{b m} T_{a k} g\right\}_{m \in \mathbb{Z}}, k \in \mathbb{Z}$, represent the signal $f$ at time $x$. Phrased differently, for each $k \in \mathbb{Z}$, the Fourier-like system $\left\{M_{b m} T_{a k} g\right\}_{m \in \mathbb{Z}}$ has an overlap of length $L / 2$ with $\left\{M_{b m} T_{a(k-1)} g\right\}_{m \in \mathbb{Z}}$ and with $\left\{M_{b m} T_{a(k+1)} g\right\}_{m \in \mathbb{Z}}$.

Under the 2-overlap condition $a=L / 2$, the painless construction is only applicable for redundancies $(a b)^{-1} \geq 2$ since $b \leq 1 / L$. We are interested in small redundancies $(a b)^{-1}<2$, that is, large modulations $b>1 / L$; note that, as is standard in Gabor analysis, in fact, necessary once continuity of the window $g$ is imposed, we always take $(a b)^{-1}>1$. Hence, we are interested in the redundancy range $(a b)^{-1} \in(1,2)$. However, once outside the region of painless non-orthonormal expansions, computing the canonical dual $\tilde{h}=S_{g}^{-1} g$ becomes much more cumbersome since it requires inverting the frame operator $S_{g}$, and one often resort to numerical approaches [15, 24, 23, 18, 2]. Even worse, beyond the painless case, i.e., $b>1 / L$, the canonical dual cannot have compact support; at least not if $a b \in \mathbb{Q}$. To be precise, for $a b \in \mathbb{Q}$, the canonical dual window has compact support if and only if $b \leq 1 / L$ as noted by Del Prete [12, p. 555],

For $n \in \mathbb{Z}_{\geq 0} \cup\{\infty\}$, we consider the window class of $n$ times continuously differentiable functions $g: \mathbb{R} \rightarrow \mathbb{C}$, i.e., $g \in C^{n}(\mathbb{R})$, that are supported on a closed interval $\left[x_{0}, x_{0}+L\right]\left(x_{0} \in \mathbb{R}\right)$ of length $L$ and nonzero on the open interval $\left(x_{0}, x_{0}+L\right)$. For any function $g$ in this window class, the Gabor system $\left\{M_{b m} T_{L k / 2} g\right\}_{k, m \in \mathbb{Z}}$ is a frame for $L^{2}(\mathbb{R})$ if and only if $b \in(0,2 / L)$ [6, Corollary 2.8]. The objective of our work is to characterize and construct compactly supported (alternate) dual windows $h$ of $g$ with good, even optimal, smoothness in a setting beyond the painless expansions region with arbitrarily small redundancy, but without having to invert the frame operator. The main features of our approach can be summarized as follows:

(I) It uses 2-overlap $(a=L / 2)$ and works outside the region of painless non-orthonormal expansions and with arbitrarily small redundancy $(a b)^{-1}>1$ of the Gabor frames.

(II) It provides a natural parametrization of all dual windows (via an explicit formula) with sufficiently small support, given in terms of a measurable function $z$ defined on a compact interval.

(III) It provides optimal smoothness of the dual window $h$, e.g., dual windows $h$ with the same smoothness as the original window, i.e., $g, h \in C^{n}(\mathbb{R})$. 
(IV) It yields support size of $h$ only dependent on the modulation parameter $b \in$ $(0,2 / L)$ and not on properties of $g$ (or $h$, e.g., smoothness).

\subsection{Results in the literature}

By dilation and translation of the Gabor system, we may without loss of generality take the translation parameter $a=1$, the modulation parameter $b \in(0,1)$, and $\operatorname{supp} g=[-1,1]$. Christensen, Kim and Kim [6] characterize the frame property of $\left\{M_{b m} T_{k} g\right\}_{m, k \in \mathbb{Z}}$ for $g \in C^{0}(\mathbb{R})$ with $\operatorname{supp} g=[-1,1]$ and finitely many zeros in $(-1,1)$. In particular, they inductively construct a continuous and compactly supported dual window once such a $g$ generates a Gabor frame $\left\{M_{b m} T_{k} g\right\}_{m, k \in \mathbb{Z}}$. While the focus in [6] is on existence questions, we aim for explicit constructions for dual windows with symmetry and higher order smoothness, albeit for a smaller class of window functions as we do not allow $g$ to have zeros inside the support.

In recent papers on so-called frame set problems $[1,8,9,13,17,20]$, the proof strategy produces a compactly supported dual window using the fiberization technique of Ron and Shen [25]. The dual windows constructed in these papers are piecewise continuous but, in general, discontinuous on the boundary of the fiber base. The constructions in [13, 17], implemented in [18], work for arbitrarily small redundancy albeit also involve solving systems of linear equations.

There exist a considerable amount of work in Gabor analysis on explicit constructions of alternate dual windows, see, e.g., $[4,10,6,7,5,19,16]$. These constructions have the desirable feature that the dual window shares or inherits many of the properties of the window, e.g., smoothness and compact support. However, the methods from the above list of works are restricted to the painless expansions region, e.g., redundancies $(a b)^{-1} \geq 2$ or even $(a b)^{-1} \geq 3$.

The construction in [19] of $C^{n}$-smooth dual windows supported on $[-1,1]$ is not guaranteed to work, however, when it does both windows are spline polynomials. Our method always works, but if $g$ is a piecewise polynomial, the dual window will in general be a piecewise rational function of polynomials. The construction of Laugesen [19] is generally limited to the painless region, but by using a trick of non-linear dilation by a soft-thresholding type function, Laugesen is able to handle smaller redundancies, i.e., $1<(a b)^{-1}<2$. However, this has the effect of making the windows constant on most of their support, which may not be desirable.

In short, our work can be seen as a continuation of $[6,7]$, but with the objective of [19] to construct smooth dual pairs of Gabor windows.

\subsection{Outline}

In Section 2 we introduce the family of dual windows. Section 3 is the main contribution with a detailed analysis of properties (smoothness, symmetry, etc.) of the dual windows. In Section 4 we present examples of the construction. 


\section{The construction of the dual windows}

For each $n \in \mathbb{Z}_{\geq 0} \cup\{\infty\}$ we define the window classes:

$V_{+}^{n}(\mathbb{R})=\left\{f \in C^{n}(\mathbb{R}): \operatorname{supp} f=[-1,1]\right.$ and $\left.\forall x \in(-1,1):|f(x)|>0, f(x) \neq-f(x+1)\right\}$.

Observe that the window classes are nested $V_{+}^{n}(\mathbb{R}) \subset V_{+}^{n-1}(\mathbb{R})$ for $n \in \mathbb{Z}_{>0}$, and that even for $g$ in the largest of these window classes $V_{+}^{0}(\mathbb{R})$, it is known [6, Corollary 2.8] that the Gabor system $\left\{M_{b m} T_{k} g\right\}_{m, k \in \mathbb{Z}}$ is a frame for $L^{2}(\mathbb{R})$ for any $b \in(0,1)$.

We now introduce compactly supported functions $h_{z}$ that will serve as dual windows of $g \in V_{+}^{0}(\mathbb{R})$; we provide some intuition behind the construction in Section 3.1. Assume $0<b<1$. Let $k_{\max } \in \mathbb{Z}_{\geq 0}$ be the largest integer strictly smaller than $b /(1-b)$, that is,

$$
k_{\max }=\max \left\{k \in \mathbb{Z}_{\geq 0}: k<\frac{b}{1-b}\right\} .
$$

Note that $k_{\max } \geq 1$ when $1 / 2<b<1$. For any $k \in\left\{0,1, \ldots, k_{\max }\right\}$ we have $k(1 / b-1)<$ 1. We define, for $k \in\left\{1,2, \ldots, k_{\max }\right\}$,

$$
[k]=\{1,2, \ldots, k\},
$$

and set $[0]=\emptyset$.

For $g \in V_{+}^{0}(\mathbb{R})$, define $\psi: \mathbb{R} \rightarrow \mathbb{C}$ by

$$
\psi(x)=\frac{1}{\sum_{n \in \mathbb{Z}} g(x+n)} \quad \text { for } x \in \mathbb{R} .
$$

By the assumptions on $g$, the 1-periodic function $\psi$ is well-defined, continuous, and satisfies

$$
c \leq|\psi(x)| \leq C \quad \text { for all } x \in \mathbb{R}
$$

for some positive, finite constants $c, C>0$. We will often consider $\psi$ as a function on $[0,1]$ with $\psi(0)=\psi(1)=1 / g(0)$ given by $\psi(x)=1 /(g(x)+g(x-1))$ for $x \in[0,1]$.

Let $z:[0,1] \rightarrow \mathbb{C}$ be a measurable function. For each $k \in\left\{0,1, \ldots, k_{\max }\right\}$ we define the following auxiliary functions:

$$
\eta_{k}(x-k)=(-1)^{k} \prod_{j \in[k]} \frac{g(x+1+j(1 / b-1))}{g(x+j(1 / b-1))}[-g(x+1) z(x+1)+b \psi(x+1)]
$$

for $x \in[-1,-k(1 / b-1)]$ and

$$
\gamma_{k}(x+k)=(-1)^{k} \prod_{j \in[k]} \frac{g(x-1-j(1 / b-1))}{g(x-j(1 / b-1))}[g(x-1) z(x)+b \psi(x)]
$$

for $x \in[k(1 / b-1), 1]$. We finally define $h_{z}: \mathbb{R} \rightarrow \mathbb{C}$ by

$$
\overline{h_{z}(x)}=\sum_{k=0}^{k_{\max }} \eta_{k}(x) \mathbf{1}_{[-k-1,-k / b]}(x)+\sum_{k=0}^{k_{\max }} \gamma_{k}(x) \mathbf{1}_{[k / b, k+1]}(x) \quad \text { for } x \in \mathbb{R} \backslash\{0\},
$$


and $\overline{h_{z}(0)}=b \psi(0)$. More explicitly, $h_{z}$ is given as:

$$
\overline{h_{z}(x)}= \begin{cases}\eta_{k}(x) & x \in[-k-1,-k / b], k=1, \ldots, k_{\max }, \\ -g(x+1) z(x+1)+b \psi(x+1) & x \in[-1,0), \\ g(x-1) z(x)+b \psi(x) & x \in[0,1], \\ \gamma_{k}(x) & x \in[k / b, k+1], k=1, \ldots, k_{\max }, \\ 0 & \text { otherwise. }\end{cases}
$$

We remark that the function $\gamma_{k}(\cdot+k)$ is indeed well-defined on $[k(1 / b-1), 1]$ since the product $\prod_{j \in[k]} \frac{1}{g(\cdot-j(1 / b-1))}$ is well-defined on $(-1+k(1 / b-1), 1 / b)$ and $[k(1 / b-1), 1] \subset$ $(-1+k(1 / b-1), 1 / b)$. Note also that the product $\prod_{j \in[k]} g(\cdot-1-j(1 / b-1))$ has support on $[k(1 / b-1), 1+1 / b]$. A similar consideration shows that $\eta_{k}$ is well-defined on its domain.

The function $h_{z}$ defined in (2.2) has compact support in $\left[-k_{\max }-1, k_{\max }+1\right]$. More precisely,

$$
\operatorname{supp} h_{z} \subset \bigcup_{k=1}^{k_{\max }}[-k-1,-k / b] \cup[-1,1] \cup \bigcup_{k=1}^{k_{\max }}[k / b, k+1] .
$$

The function $h_{z}$ is piecewise defined with $\cup_{k=0}^{k_{\max }}\{ \pm k / b, \pm(k+1)\}$ being the seam points of $h_{z}$. The seam point $x=0$ is special as it is the only seam point, where two nonzero functions in the definition of $h_{z}$ meet. In all other seam points, i.e., $x \neq 0$, the function $h_{z}$ is zero on one side of the seam points.

Remark 2.1. For later use, we remark that Christensen, Kim, and Kim [6] consider dual windows $h \in C^{0}(\mathbb{R})$ of $g \in V_{+}^{0}(\mathbb{R})$ with compact support supp $h \subset[-N, N]$, where $N:=\max \left\{n \in \mathbb{Z}_{>0}: n \leq b /(1-b)\right\}+1$ and $b \geq 1 / 2$. When $b /(1-b) \notin \mathbb{Z}_{>0}$, then $N=k_{\max }+1$, and the support $\operatorname{supp} h \subset[-N, N]$ corresponds to the support of $h_{z}$ in (2.3). On the other hand, if $b /(1-b) \in \mathbb{Z}_{>0}$, there is a mismatch in the support relation as $N=k_{\max }+2$. However, in case $b /(1-b) \in \mathbb{Z}_{>0}$ we have $(N-1) / b=N$, and the interval $[(N-1) / b, N]$ collapses to a point $\{N\}$. As a consequence, we can, in any case, use $N:=k_{\max }+1$ when applying results from [6].

\section{Properties of the dual windows}

We first prove that $h_{z}$ indeed is a dual window of $g$ as soon as $\left\{M_{b m} T_{k} h_{z}\right\}_{m, k \in \mathbb{Z}}$ is a Bessel system in Section 3.1. In Section 3.2 we show that the chosen parametrization $z \mapsto h_{z}$ has several desirable properties. In Section 3.3 we show how to construct smooth dual windows $h \in C^{n}(\mathbb{R})$ for any $g \in V_{+}^{n}(\mathbb{R})$ and $n \in \mathbb{Z}_{\geq 0} \cup\{\infty\}$. In Section 3.4 we discuss optimal smoothness of dual windows and show that the results in Section 3.3, in general, are optimal. In Section 3.5 we minimize the support length of the dual window in the sense of [7] while preserving the optimal smoothness.

However, we first introduce some notation used below. We use $f\left(x_{0}^{+}\right)$to denote the one-sided limit from the right $\lim _{x \searrow_{0}} f(x)$ and similarly $f\left(x_{0}^{-}\right)$to denote the one-sided limit from the $\operatorname{left} \lim _{x \nearrow x_{0}} f(x)$. For $n \in \mathbb{Z}_{\geq 0}$, we let $D^{n}[f]$ and $f^{(n)}$ denote the $n$th 
derivative of a function $f: \mathbb{R} \rightarrow \mathbb{C}$, with the convention $f(x)=D^{0}[f](x)=f^{(0)}(x)$. We will repeatedly use the (general) Leibniz rule for differentiation of products:

$$
(f g)^{(n)}(x)=\sum_{\ell=0}^{n}\left(\begin{array}{l}
n \\
\ell
\end{array}\right) f^{(n-\ell)}(x) g^{(\ell)}(x),
$$

where $\left(\begin{array}{l}n \\ \ell\end{array}\right)=\frac{n !}{\ell !(n-\ell) !}$ is the binomial coefficient.

A function $f:[c, d] \rightarrow \mathbb{C}$ is said to be piecewise $C^{n}$ if there exists a finite subdivision $\left\{x_{0}, \ldots, x_{k}\right\}$ of $[c, d], x_{0}=c, x_{k}=d$ such that $f$ is $C^{n}$ on $\left[x_{i-1}, x_{i}\right]$, the derivatives at $x_{i-1}$ understood as right-handed and the derivatives at $x_{i}$ understood as left-handed, for every $i \in\{1, \ldots, k\}$. Hence, if $f$ is piecewise $C^{n}$, the one-sided limits of $f^{(m)}$ exists everywhere for all $m=0, \ldots, n$, but the left and right limits may differ in a finite number of points. The definition of piecewise $C^{n}$ functions can be extended to functions on $\mathbb{R}$, we will, however, only need it for compactly supported functions, where the modification is obvious.

\subsection{Duality}

The following duality condition for two Gabor systems by Ron and Shen $[25,26]$ is central to our work; we use the formulation due to Janssen[14].

Theorem 3.1 ([25]). Let $b>0$ and $g, h \in L^{2}(\mathbb{R})$. Suppose $\left\{M_{b m} T_{k} g\right\}_{m, k \in \mathbb{Z}}$ and $\left\{M_{b m} T_{k} h\right\}_{m, k \in \mathbb{Z}}$ are Bessel sequences. Then $\left\{M_{b m} T_{k} g\right\}_{m, k \in \mathbb{Z}}$ and $\left\{M_{b m} T_{k} h\right\}_{m, k \in \mathbb{Z}}$ are dual frames for $L^{2}(\mathbb{R})$, if and only if, for all $k \in \mathbb{Z}$,

$$
\sum_{n \in \mathbb{Z}} g(x-k / b+n) \overline{h(x+n)}=\delta_{0, k} b \quad \text { for a.e. } x \in \mathbb{R} .
$$

Since the infinite series in (3.2) is 1-periodic, it suffices to verify (3.2) on any interval of length one. Furthermore, for supp $g \subset[-1,1]$, the duality conditions (3.2) become, for $k \neq 0$,

$$
g(x-k / b) \overline{h(x)}+g(x-k / b-1) \overline{h(x-1)}=0 \quad \text { for a.e. } x \in[k / b, k / b+1]
$$

and, for $k=0$,

$$
g(x) \overline{h(x)}+g(x-1) \overline{h(x-1)}=b \quad \text { for a.e. } x \in[0,1] .
$$

The following theorem shows that $g$ and $h:=h_{z}$, defined in (2.2), indeed satisfy (3.2) for all $k \in \mathbb{Z}$ and, hence, that $h_{z}$ is a convenient representation of dual windows of $g$.

Theorem 3.2. Let $b \in(0,1)$, let $g \in V_{+}^{0}(\mathbb{R})$, and let $z:[0,1] \rightarrow \mathbb{C}$ be a measurable function. Then $g$ and $h_{z}$ satisfy the characterizing equations (3.2) for all $k \in \mathbb{Z}$. Hence, if $\left\{M_{b m} T_{k} h_{z}\right\}_{m, k \in \mathbb{Z}}$ is a Bessel sequence, e.g., if $h_{z} \in L^{\infty}(\mathbb{R})$, then $\left\{M_{b m} T_{k} g\right\}_{m, k \in \mathbb{Z}}$ and $\left\{M_{b m} T_{k} h_{z}\right\}_{m, k \in \mathbb{Z}}$ are dual frames for $L^{2}(\mathbb{R})$. 
Before proving Theorem 3.2 let us provide some intuition behind the definition of $h_{z}$ as this also illustrates the proof, i.e., why $g$ and $h_{z}$ satisfy (3.2) for all $k \in \mathbb{Z}$. The construction starts with a function $h \in L^{2}(\mathbb{R})$ that is arbitrary a.e. on $[0,1]$ and zero otherwise. One then inductively modifies the definition of $h$ on $\mathbb{R} \backslash[0,1]$ to satisfy (3.2) for increasing values of $|k|=0,1, \ldots, k_{\max }$.

We first modify $h$ on $[-1,0]$ so that the condition (3.2) for $k=0$ holds. Since $1 / b>1$ and the support length for $g$ is 2 , the only conditions $(3.2), k \neq 0$, that could be affected by this modification are the conditions for $k= \pm 1$. Hence, we turn our attention to these two conditions.

In case $b \leq 1 / 2$, there is no overlap between $h$ and $g(\cdot \pm 1 / b)$, and $g$ and $h$ already satisfy (3.2) for all $k \in \mathbb{Z}$, and we are done. In case $b>1 / 2$, there will be overlap between (the support of) $h$ and $g(\cdot \pm 1 / b)$, each overlap less than one in length since $1 / b>1$. In order to satisfy (3.2) for $k= \pm 1$, we modify $h$ on intervals of the same length as the overlap but shifted by $\mp 1$ for the overlap of $h$ with $g(\cdot \pm 1 / b)$. That is, we modify $h$ on $[-2,1-1 / b]$ and $[-1+1 / b, 2]$ so that $(3.2)$ holds for $k=1$ and $k=-1$, respectively.

The functions $g$ and $h$ now satisfy (3.2) for $k=0, \pm 1$ (as well as for $|k| \geq 3$ ), hence we check if $h$ overlaps with either of the functions $g(x \pm 2 / b)$. If there is no overlap, we are done as $g$ and $h$ already satisfy (3.2) for all $k \in \mathbb{Z}$; Figure 1 illustrates this situation.

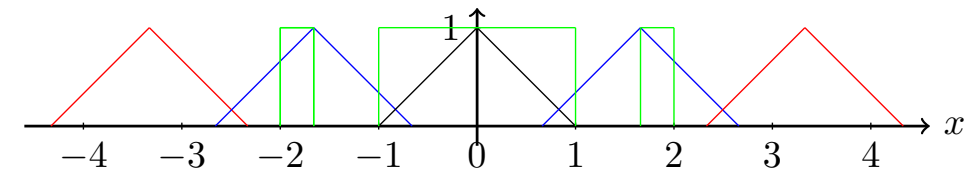

Figure 1: Illustration of the inductive modification of $h$ for $g(x)=\max (0,1-|x|)$ (black). The graphs of $g(\cdot \pm 1 / b)$ are shown in blue, and $g(\cdot \pm 2 / b)$ in red, and $\mathbf{1}_{(\operatorname{supp} h)}$ in green.

If there is an overlap, then the procedure is continued so that (3.2) also holds for $k= \pm 2$. We keep moving outwards from the origo $x=0$ until the next translates $g(\cdot \pm k / b)$ no longer overlap with the function $h$. Since $1 / b>1$, we know that the process will terminate as the overlap size shrinks in each iteration. In fact, $k_{\max }$ is exactly defined to make the process terminate at $|k|=k_{\max }$. The fact that $1 / b>1$ also guarantees that no more than two of the translates $g(\cdot-k / b)$ for $k \in \mathbb{Z}$ will overlap. This is crucial for the inductive procedure as it implies that at most one equation (3.2) is affected when modifying $h$ on an overlap interval.

Proof of Theorem 3.2. First, we check that (3.2) holds for $k=0$. Since

$$
g(x) \overline{h_{z}(x)}= \begin{cases}g(x)[-g(x+1) z(x+1)+b \psi(x+1)] & \text { a.e. } x \in[-1,0) \\ g(x)[g(x-1) z(x)+b \psi(x)] & \text { a.e. } x \in[0,1) \\ 0 & \text { otherwise, }\end{cases}
$$


it follows that, for a.e. $x \in[0,1)$,

$$
\begin{aligned}
\sum_{n \in \mathbb{Z}} g(x+n) \overline{h_{z}(x+n)}= & -g(x-1) g(x) z(x)+g(x-1) b \psi(x) \\
& +g(x) g(x-1) z(x)+g(x) b \psi(x) \\
= & b \psi(x)(g(x-1)+g(x))=b,
\end{aligned}
$$

where the final equality follows from the definition of $\psi(x)$. This shows that (3.2) holds for $k=0$.

For $|k| \geq k_{\max }+1$ the functions $g(\cdot-k / b)$ and $h_{z}$ have disjoint support. This follows from $(2.3)$ and the fact that $k_{\max }+1 \leq-1+\left(k_{\max }+1\right) / b$. Consequently, equation (3.2) holds for $|k| \geq k_{\max }+1$.

For $k \in\left\{1, \ldots, k_{\max }\right\}$ we verify $(3.2)$ on $[k, k+1)$. We first compute

$$
g(x-k / b) \overline{h_{z}(x)}= \begin{cases}g(x-k / b) \gamma_{k-1}(x) & \text { a.e. } x \in[k / b-1, k), \\ g(x-k / b) \gamma_{k}(x) & \text { a.e. } x \in[k / b, k+1), \\ 0 & \text { otherwise. }\end{cases}
$$

Hence, for a.e. $x \in[k, k / b)$, it follows trivially that

$$
\sum_{n \in \mathbb{Z}} g(x-k / b+n) \overline{h_{z}(x+n)}=0 .
$$

On the other hand, for a.e. $x \in[k / b, k+1)$, we have

$$
\sum_{n \in \mathbb{Z}} g\left(x-\frac{k}{b}+n\right) \overline{h_{z}(x+n)}=g\left(x-\frac{k}{b}\right) \gamma_{k}(x)+g\left(x-\frac{k}{b}-1\right) \gamma_{k-1}(x-1) .
$$

We focus on the first term of the right hand side, which, by definition, is given as:

$g\left(x-\frac{k}{b}\right) \gamma_{k}(x)=g\left(x-\frac{k}{b}\right)(-1)^{k} \prod_{j \in[k]} \frac{g\left(x-k-1-j\left(\frac{1}{b}-1\right)\right)}{g\left(x-k-j\left(\frac{1}{b}-1\right)\right)}[g(x-k-1) z(x-k)+b \psi(x-k)]$.

Since

$$
\prod_{j \in[k]} \frac{g\left(x-k-1-j\left(\frac{1}{b}-1\right)\right)}{g\left(x-k-j\left(\frac{1}{b}-1\right)\right)}=\frac{g\left(x-\frac{k}{b}-1\right)}{g\left(x-\frac{k}{b}\right)} \prod_{j \in[k-1]} \frac{g\left(x-k-1-j\left(\frac{1}{b}-1\right)\right)}{g\left(x-k-j\left(\frac{1}{b}-1\right)\right)},
$$

we can rewrite $g\left(x-\frac{k}{b}\right) \gamma_{k}(x)$ as follows:

$$
\begin{aligned}
g\left(x-\frac{k}{b}\right) \gamma_{k}(x) & =g\left(x-\frac{k}{b}-1\right)(-1)^{k} \prod_{j \in[k-1]} \frac{g\left(x-k-1-j\left(\frac{1}{b}-1\right)\right)}{g\left(x-k-j\left(\frac{1}{b}-1\right)\right)} \\
\cdot & {[g(x-k-1) z(x-k)+b \psi(x-k)] } \\
& =-g\left(x-\frac{k}{b}-1\right) \gamma_{k-1}(x-1) .
\end{aligned}
$$


By inserting this back into (3.3), we obtain, for a.e. $x \in[k / b, k+1)$,

$\sum_{n \in \mathbb{Z}} g\left(x-\frac{k}{b}+n\right) \overline{h_{z}(x+n)}=-g\left(x-\frac{k}{b}-1\right) \gamma_{k-1}(x-1)+g\left(x-\frac{k}{b}-1\right) \gamma_{k-1}(x-1)=0$,

which verifies $(3.2)$ for $k \in\left\{1, \ldots, k_{\max }\right\}$.

The calculations for $k \in\left\{-k_{\max }, \ldots,-1\right\}$ are similar to the above calculations, hence we leave this case for the reader.

The next result, Lemma 3.3, shows that not only is $h_{z}$ a convenient expression of dual windows of $g \in V_{+}^{0}(\mathbb{R})$, it is in fact a parametrization by 1-periodic measurable functions $z$ of all dual windows with sufficiently small support. The structure of the proof of Lemma 3.3 is somewhat similar to the proof of Lemma 3.3 in [6]. As the two results are quite different, we give the proof of Lemma 3.3.

Lemma 3.3 (Parametrization of all compactly supported dual windows). Let $b \in(0,1)$ and $g \in V_{+}^{0}(\mathbb{R})$. Suppose $h \in L^{2}(\mathbb{R})$ has compact support in $\left[-k_{\max }-1, k_{\max }+1\right]$. If $\left\{M_{b m} T_{k} g\right\}_{m, k \in \mathbb{Z}}$ and $\left\{M_{b m} T_{k} h\right\}_{m, k \in \mathbb{Z}}$ are dual frames, then $h=h_{z}$ for some measurable function $z:[0,1] \rightarrow \mathbb{C}$.

Proof. Let $h \in L^{2}(\mathbb{R})$ be a dual window of $g$ with supp $h \subset\left[-k_{\max }-1, k_{\max }+1\right]$. Lemma 3.2 in [6] says, see Remark 2.1, that

$$
\operatorname{supp} h \subset \bigcup_{k=1}^{k_{\max }}[-k-1,-k / b] \cup[-1,1] \cup \bigcup_{k=1}^{k_{\max }}[k / b, k+1] .
$$

Define a measurable function $z$ on $[0,1]$ by:

$$
z(x)=\frac{\overline{h(x)}-b \psi(x)}{g(x-1)} \quad \text { a.e. } x \in(0,1) .
$$

This definition gives immediately that $h(x)=h_{z}(x)$ for a.e. $x \in[0,1]$.

By (3.2) with $k=0$, we have for a.e. $x \in(-1,0)$

$$
\overline{h(x)}=\frac{-g(x+1) \overline{h(x+1)}+b}{g(x)}
$$

We continue, using that $h(x)=h_{z}(x)$ for a.e. $x \in[0,1]$,

$$
\begin{aligned}
\overline{h(x)} & =\frac{-g(x+1)[-g(x) z(x+1)+b \psi(x+1)]+b}{g(x)} \\
& =-g(x+1) z(x+1)+b \psi(x+1)=\overline{h_{z}(x)} \quad \text { for } x \in(-1,1) .
\end{aligned}
$$

Thus, also $h(x)=h_{z}(x)$ for a.e. $x \in[-1,1]$.

We will complete the proof by induction, showing that

$$
h(x)=h_{z}(x) \quad \text { for a.e. } x \in \bigcup_{k=1}^{k_{0}}[-k-1,-k / b] \cup[-1,1] \cup \bigcup_{k=1}^{k_{0}}[k / b, k+1] .
$$


holds for all integers $k_{0}$ in $1 \leq k_{0} \leq k_{\max }$. The base case $k_{0}=0$ was verified above so we only have to show the induction step $k_{0}-1 \rightarrow k_{0}$.

We first consider $x>0$. By induction hypothesis, we have $\overline{h(x)}=\overline{h_{z}(x)}=\gamma_{k_{0}-1}(x)$ for a.e. $x \in\left[\left(k_{0}-1\right) / b, k_{0}\right]$. We aim to prove $\overline{h(x)}=\gamma_{k_{0}}(x)$ for a.e. $x \in\left[k_{0} / b, k_{0}+1\right]$, or rather $\overline{h(x+1)}=\gamma_{k_{0}}(x+1)$ for a.e. $x \in\left[k_{0} / b-1, k_{0}\right]$. Since $\left[k_{0} / b-1, k_{0}\right] \subset$ $\left[k_{0} / b-1, k_{0} / b\right]$, it follows by (3.2) for $k=k_{0}$ :

$$
\overline{h(x+1)}=-\frac{g\left(x-k_{0} / b\right)}{g\left(x-k_{0} / b+1\right)} \cdot \overline{h(x)}
$$

for a.e. $x \in\left[k_{0} / b-1, k_{0}\right]$. Since also $\left[k_{0} / b-1, k_{0}\right] \subset\left[\left(k_{0}-1\right) / b, k_{0}\right]$, using the induction hypothesis yields further:

$$
\overline{h(x+1)}=-\frac{g\left(x-k_{0}-k_{0}(1 / b-1)\right)}{g\left(x-k_{0}+1-k_{0}(1 / b-1)\right)} \cdot \gamma_{k_{0}-1}(x)=\gamma_{k_{0}}(x+1)=\overline{h_{z}(x+1)}
$$

for a.e. $x \in\left[k_{0} / b-1, k_{0}\right]$. The argument for $x<0$ is similar, hence we omit it.

Obviously, there are choices of an unbounded function $z$ that leads to $h_{z}$ not generating a Gabor Bessel sequence in $L^{2}(\mathbb{R})$, e.g., if $h_{z} \notin L^{2}(\mathbb{R})$, in which case $\left\{M_{b m} T_{k} g\right\}_{m, k \in \mathbb{Z}}$ and $\left\{M_{b m} T_{k} h_{z}\right\}_{m, k \in \mathbb{Z}}$ are not dual frames. This is not contradicting Lemma 3.3.

Lemma 3.3 should be compared to the well-known parametrization of all dual windows $h$ by functions $\varphi$ generating Bessel Gabor systems, see, e.g., [3, Proposition 12.3.6]; the parametrization formula (3.4) is due to $\mathrm{Li}[22]$ and reads:

$$
h=S_{g}^{-1} g+\varphi-\sum_{m, k \in \mathbb{Z}}\left\langle S^{-1} g, M_{b m} T_{a k} g\right\rangle M_{b m} T_{a k} \varphi .
$$

The parametrization by Bessel generators $\varphi$ is not injective nor explicit as one needs to compute both $S_{g}^{-1} g$ and an infinite series. On the other hand, the mapping $z \mapsto$ $h_{z}(z$ measurable on $[0,1])$ is injective and its range contains all dual windows with compact support on $\left[-k_{\max }-1, k_{\max }+1\right]$. Moreover, by Lemma 3.4 below, the mapping $L^{\infty}([0,1]) \ni z \mapsto h_{z}$ is a bijective and explicit parametrization of all bounded dual windows with sufficiently small support, i.e., $\operatorname{supp} h \subset\left[-k_{\max }-1, k_{\max }+1\right]$. Of course, the parametrization in Lemma 3.3 only works for our setting, in particular, only under the 2-overlap condition, i.e., $a=L / 2$, while formula (3.4) works for all Gabor frames.

\subsection{Basic properties: symmetry, boundedness, and continuity}

The next two lemmas show that the chosen parametrization $z \mapsto h_{z}$ is rather natural. Indeed, both symmetry and boundedness properties of $g$ and $z$ are transferred to $h_{z}$.

Lemma 3.4 (Boundedness). Let $b \in(0,1)$. Suppose $g \in V_{+}^{0}(\mathbb{R})$. Then $h_{z} \in L^{\infty}(\mathbb{R})$ if and only if $z \in L^{\infty}([0,1])$. 
Proof. To show the "only if"-implication, note that for a.e. $x \in[0,1]$, we have $\overline{h_{z}(x)}=$ $g(x-1) z(x)+b \psi(x)$. Since $|g(x)|$ is bounded (on $\mathbb{R}$ ) and positive on $(-1,1)$, it follows that if $h_{z}$ is bounded on $[0,1]$, then so is $z$ on $[c, 1]$ for any $c>0$. Using the boundedness of $h_{z}$ on $[-1,0]$ leads to the conclusion that $z$ is bounded on $[0,1-c]$ for any $c>0$.

For the converse assertion, let $k \in\left\{1, \ldots, k_{\max }\right\}$. Once we argue for the boundedness of $\gamma_{k}$ and $\eta_{k}$ on $[k / b, k+1]$ and $[-k-1,-k / b]$, respectively, the assertion is clear. We only consider $\gamma_{k}$ as the argument of $\eta_{k}$ is similar. Now, to argue for boundedness of $\gamma_{k}$, it suffices to show that the product

$$
\prod_{j \in[k]} \frac{1}{g(x-j(1 / b-1))} \quad \text { for } x \in[k(1 / b-1), 1]
$$

is bounded. For all $j \in[k]$ and all $x \in[k(1 / b-1), 1]$ we have

$$
0=k\left(\frac{1}{b}-1\right)-k\left(\frac{1}{b}-1\right) \leq x-j\left(\frac{1}{b}-1\right) \leq 2-\frac{1}{b}<1
$$

Let $c$ denote the positive minimum of the continuous function $|g|$ on the compact interval $[-2+1 / b, 2-1 / b]$. Then

$$
\sup _{x \in[k(1 / b-1), 1]} \prod_{j \in[k]}\left|\frac{1}{g(x-j(1 / b-1))}\right| \leq k / c,
$$

which completes the proof.

Lemma 3.5 (Symmetry). Let $b \in(0,1)$. Suppose $g \in V_{+}^{0}(\mathbb{R})$ is even. Then $h_{z}$ is even if and only if $z$ is antisymmetric around $x=1 / 2$, i.e., $z(x)=-z(1-x)$ for a.e. $x \in[0,1 / 2]$.

Proof. Assume first $z(x)=-z(1-x)$ for a.e. $x \in \mathbb{R}$. If $g$ is even, then so is $\psi$, and it follows by straightforward verification in the definition $(2.2)$ that $h_{z}(x)=h_{z}(-x)$ holds for a.e. $x \in \mathbb{R}$.

On the other hand, if $h_{z}$ is even, then using the definition of $h_{z}$ on $[-1,1]$, it follows easily that $z$ is antisymmetric around $x=1 / 2$.

The last lemma of this subsection characterizes continuity of the dual windows $h_{z}$ in terms of easy verifiable conditions on $z$.

Lemma 3.6 (Continuity). Let $b \in(0,1)$. Suppose $g \in V_{+}^{0}(\mathbb{R})$. Then $h_{z} \in C^{0}(\mathbb{R})$ if and only if $z:[0,1] \rightarrow \mathbb{C}$ is a continuous function satisfying

$$
z(0)=\frac{b \psi(0)}{g(0)}=\frac{b}{g(0)^{2}}
$$

and

$$
z(1)=-\frac{b \psi(1)}{g(0)}=-\frac{b}{g(0)^{2}}
$$


Proof. Suppose first that $h_{z} \in C^{0}(\mathbb{R})$. Then since for $x \in(0,1]$, we have $g(x-1) \neq 0$ and $\overline{h_{z}(x)}=g(x-1) z(x)+b \psi(x)$ with $h_{z}, g, \psi \in C^{0}(\mathbb{R})$, it follows that $z$ is continuous on $(0,1]$. Continuity of $z$ at $x=0$, i.e., existence of $\lim _{x \backslash 0} z(x)$, follows by similar considerations of $\left.h_{z}\right|_{[-1,0)}$. By continuity of $h_{z}$ and $(2.3)$, we have $h_{z}( \pm 1)=0$. Hence,

$$
0=\overline{h_{z}(-1)}=-g(0) z(0)+b \psi(0) \quad \text { and } \quad 0=\overline{h_{z}(1)}=g(0) z(1)+b \psi(1)
$$

which shows the "only if"-implication.

To show the other implication, we have to work a little harder. On the open set

$$
\mathbb{R} \backslash\left(\bigcup_{k=0}^{k_{\max }}\{ \pm k / b, \pm(k+1)\}\right)
$$

the function $h_{z}$ is continuous since it is a sum and product of continuous functions on this set.

To show continuity at the seam points $\cup_{k=0}^{k_{\max }}\{ \pm k / b, \pm(k+1)\}$, it suffices to show

$$
h_{z}\left(0^{-}\right)=h_{z}\left(0^{+}\right), \quad h_{z}( \pm k / b)=0, k \neq 0, \quad \text { and } \quad h_{z}( \pm(k+1))=0
$$

for $k \in\left\{0,1, \ldots, k_{\max }\right\}$. Fix $k \in\left\{0,1, \ldots, k_{\max }\right\}$. We first focus on the seam points in $(-\infty,-1]$. For $x=-k-1$ we immediately have

$$
\overline{h_{z}(-k-1)}=(-1)^{k} \prod_{j \in[k]} \frac{g(j(1 / b-1))}{g(-1+j(1 / b-1))}[-g(0) z(0)+b \psi(0)]=0
$$

as the expression in the square brackets is zero by our assumption on $z(0)$ in (3.5).

For $x=-k / b, k \neq 0$, we note that $\overline{h_{z}(-k / b)}$, by definition, contains the product

$$
\prod_{j \in[k]} \frac{g(-k / b+k+1+j(1 / b-1))}{g(-k / b+k+j(1 / b-1))}
$$

as a factor. Further, since $k \in[k]$, this product has $\frac{g(-k / b+k+1+k(1 / b-1))}{g(-k / b+k+k(1 / b-1))}=\frac{g(1)}{g(0)}$ as one of its factors. Since $g(1)=0$ by the support and continuity assumption $g \in V_{+}^{0}(\mathbb{R})$, it follows that $h_{z}(-k / b)=0$ for $k=1, \ldots, k_{\max }$.

Now, we consider seam points in $[1, \infty)$. For $x=k+1$ we have by $(3.6)$ that

$$
\overline{h_{z}(k+1)}=(-1)^{k} \prod_{j \in[k]} \frac{g(-j(1 / b-1))}{g(1-j(1 / b-1))}[g(0) z(1)+b \psi(1)]=0 .
$$

To see $h_{z}(k / b)=0$ we note that the product defining $\overline{h_{z}(k / b)}$ contains the factor $g(-1) / g(0)$ which is zero due to the assumption $g \in V_{+}^{0}(\mathbb{R})$.

Finally, we show continuity of $h_{z}(x)$ at $x=0$. However, this follows readily by considering the two one-sided limits $x \nearrow 0$ and $x \searrow 0$ of $h_{z}(x)$ :

$$
\overline{h_{z}\left(0^{-}\right)}=-g(1) z(1)+b \psi(1)=b \psi(1)=b / g(0)
$$

and

$$
\overline{h_{z}\left(0^{+}\right)}=g(-1) z(0)+b \psi(0)=b \psi(0)=b / g(0),
$$

respectively. 


\subsection{Higher order smoothness}

The main result of this section, Theorem 3.8, characterizes $C^{n}$-smoothness of $h_{z}$ in terms of conditions on $z$. As these conditions involves derivatives of $\psi$, more precisely, $\psi^{(m)}(0)$, the next lemma shows how to operate with this condition.

Lemma 3.7. Let $n \in \mathbb{Z}_{>0}$, and let $g \in V_{+}^{n}(\mathbb{R})$. Then $\psi:=\frac{1}{\sum_{n \in \mathbb{Z}} g(\cdot+n)} \in C^{n}(\mathbb{R})$ and

$$
\psi^{(n)}(0)=\sum_{\mathbf{m} \in M} \frac{n !}{m_{1} ! m_{2} ! \cdots m_{n} !} \frac{(-1)^{m_{1}+\cdots+m_{n}}\left(m_{1}+\cdots+m_{n}\right) !}{g(0)^{m_{1}+\cdots+m_{n}+1}} \prod_{j=1}^{n}\left(\frac{g^{(j)}(0)}{j !}\right)^{m_{j}}
$$

where $M:=\left\{\left(m_{1}, \ldots, m_{n}\right) \in\left(\mathbb{Z}_{\geq 0}\right)^{n} \mid 1 \cdot m_{1}+2 \cdot m_{2}+\cdots+n \cdot m_{n}=n\right\}$.

Proof. Since $g \in V_{+}^{n}(\mathbb{R})$, the sum $\sum_{n \in \mathbb{Z}} g(\cdot+n)$ is in $C^{n}(\mathbb{R})$ and is bounded below by a positive constant. Hence, since the mapping $x \mapsto 1 / x$ is $C^{\infty}$ on $(0, \infty)$, we have $\psi \in C^{n}(\mathbb{R})$.

As usual, we consider $\psi$ on $[0,1]$ where the function is given by $x \mapsto 1 /(g(x)+g(x-$ 1)). We will use the following version of Faà di Bruno's formula:

$$
\frac{d^{n}}{d x^{n}} f(h(x))=\sum_{\mathbf{m} \in M} \frac{n !}{m_{1} ! m_{2} ! \cdots m_{n} !} f^{\left(m_{1}+\cdots+m_{n}\right)}(h(x)) \prod_{j=1}^{n}\left(\frac{h^{(j)}(x)}{j !}\right)^{m_{j}} .
$$

Taking $f(x)=\frac{1}{x}$ and $h(x)=g(x)+g(x-1)$ in Faà di Bruno's formula yields

$$
\begin{aligned}
\psi^{(n)}(0) & =\sum_{\mathbf{m} \in M} \frac{n !}{m_{1} ! m_{2} ! \cdots m_{n} !} \frac{(-1)^{m_{1}+\cdots+m_{n}}\left(m_{1}+\cdots+m_{n}\right) !}{(g(0)+g(-1))^{m_{1}+\cdots+m_{n}+1}} \prod_{j=1}^{n}\left(\frac{g^{(j)}(0)+g^{(j)}(-1)}{j !}\right)^{m_{j}} \\
& =\sum_{\mathbf{m} \in M} \frac{n !}{m_{1} ! m_{2} ! \cdots m_{n} !} \frac{(-1)^{m_{1}+\cdots+m_{n}}\left(m_{1}+\cdots+m_{n}\right) !}{g(0)^{m_{1}+\cdots+m_{n}+1}} \prod_{j=1}^{n}\left(\frac{g^{(j)}(0)}{j !}\right)^{m_{j}},
\end{aligned}
$$

using $d^{m}\left(x^{-1}\right) / d x^{m}=(-1)^{m} m ! x^{-(m+1)}$ for $m \in \mathbb{Z}_{>0}$ in the first equality and $g^{(\ell)}(-1)=$ 0 for $\ell=0,1, \ldots, n$ in the second.

Example 3.1. We illustrate the computation of Lemma 3.7 for $n=1,2$. For $n=1$, since $M=\{1\}$, formula (3.7) simply yields

$$
\psi^{(1)}(0)=-\frac{g^{(1)}(0)}{g(0)^{2}} .
$$

For $n=2$, we have $M=\{(2,0),(0,1)\}$, whereby

$$
\psi^{(2)}(0)=2 \frac{g^{(1)}(0)^{2}}{g(0)^{3}}-\frac{g^{(2)}(0)}{g(0)^{2}} .
$$

Theorem 3.8. Let $n \in \mathbb{Z}_{>0} \cup\{\infty\}$, and let $g \in V_{+}^{n}(\mathbb{R})$. The following assertions are equivalent: 
(i) $z \in C^{n}([0,1])$ satisfies (3.5), (3.6), and, for each $m=1, \ldots, n$,

$$
z^{(m)}(0)=-\sum_{\ell=1}^{m}\left(\begin{array}{c}
m \\
\ell
\end{array}\right) \frac{g^{(\ell)}(0)}{g(0)} z^{(m-\ell)}(0)+b \frac{\psi^{(m)}(0)}{g(0)},
$$

and

$$
z^{(m)}(1)=-\sum_{\ell=1}^{m}\left(\begin{array}{c}
m \\
\ell
\end{array}\right) \frac{g^{(\ell)}(0)}{g(0)} z^{(m-\ell)}(1)-b \frac{\psi^{(m)}(0)}{g(0)} .
$$

(ii) $h_{z} \in C^{n}(\mathbb{R})$.

Proof. We first prove the assertion (i) $\Rightarrow$ (ii). Consider the open set

$$
J:=\mathbb{R} \backslash\left(\bigcup_{k=0}^{k_{\max }}\{ \pm k / b, \pm(k+1)\}\right) .
$$

The function $\left.h_{z}\right|_{J}$ is in $C^{n}(J)$ since it is a sum and product of $C^{n}(J)$ functions.

To prove $C^{n}$-smoothness at the seam points $\cup_{k=0}^{k_{\max }}\{ \pm k / b, \pm(k+1)\}$, we need to show that

$$
h_{z}^{(m)}\left(0^{-}\right)=h_{z}^{(m)}\left(0^{+}\right), \quad h_{z}^{(m)}( \pm k / b)=0, k \neq 0, \quad \text { and } \quad h_{z}^{(m)}( \pm(k+1))=0
$$

for $m=1, \ldots, n$ and $k \in\left\{0,1, \ldots, k_{\max }\right\}$. The proof of this is split into the three cases:

$$
x=0, \quad x \in \bigcup_{k=0}^{k_{\max }}\{ \pm k / b\} \quad \text { and } \quad x \in \bigcup_{k=0}^{k_{\max }}\{ \pm(k+1)\} .
$$

However, we first note that $g \in V_{+}^{n}(\mathbb{R})$ implies $g^{(m)}( \pm 1)=0$ for $m=0, \ldots, n$,

$C^{n}$-smoothness at $x=0$. For $x \in[0,1]$

$$
\overline{h_{z}^{(m)}(x)}=\sum_{\ell=0}^{m}\left(\begin{array}{c}
m \\
\ell
\end{array}\right) g^{(\ell)}(x+1) z^{(m-\ell)}(x+1)+b \psi^{(m)}(x) .
$$

Since $g^{(m)}(1)=0$ for $m=0, \ldots, n$, it is readily seen that

$$
\begin{aligned}
\overline{h_{z}^{(m)}\left(0^{-}\right)} & =\sum_{\ell=0}^{m}\left(\begin{array}{c}
m \\
\ell
\end{array}\right) g^{(\ell)}(1) z^{(m-\ell)}(1)+b \psi^{(m)}(0) \\
& =b \psi^{(m)}(0)
\end{aligned}
$$

for all $m \leq n$.

Similarly, by considering $x \in[-1,0)$, we see that

$$
\overline{h_{z}^{(m)}\left(0^{+}\right)}=-\sum_{\ell=0}^{m}\left(\begin{array}{c}
m \\
\ell
\end{array}\right) g^{(\ell)}(-1) z^{(m-\ell)}(0)+b \psi^{(m)}(1)=b \psi^{(m)}(0),
$$


where the last equality follows from $g^{(m)}(-1)=0$ for $m=0, \ldots, n$ and periodicity of $\psi$.

In the two remaining cases, we will use the following easy consequence of the Leibniz rule (3.1). If $f, g \in C^{n}(\mathbb{R})$ and $g^{(m)}\left(x_{0}\right)=0$ for $m=0,1, \ldots, n$, then $(f g)^{(m)}\left(x_{0}\right)=0$ for $m=0,1, \ldots, n$, where $f g=x \mapsto f(x) g(x)$.

$C^{n}$-smoothness at $x= \pm k / b, k=1, \ldots, k_{\max }$. We first consider $x=k / b, k=$ $1, \ldots, k_{\max }$. By rearranging the terms in the definition of the auxiliary function $\gamma_{k}(x+k)$ in $(2.1)$, we obtain

$$
\begin{aligned}
\gamma_{k}(x+k) & =g(x-1-k(1 / b-1)) \\
& \cdot \frac{(-1)^{k}}{g(x-k(1 / b-1)))} \prod_{j \in[k-1]} \frac{g(x-1-j(1 / b-1))}{g(x-j(1 / b-1))}[g(x-1) z(x)+b \psi(x)],
\end{aligned}
$$

for $x \in[k(1 / b-1), 1]$. Thus, for $x \in[k / b, k+1]$, we will consider $\gamma_{k}(x)$ a product of the functions

$$
g(x-1-k / b)
$$

and

$$
\frac{(-1)^{k}}{g(x-k / b)} \prod_{j \in[k-1]} \frac{g(x-k-1-j(1 / b-1))}{g(x-k-j(1 / b-1))}[g(x-k-1) z(x-k)+b \psi(x-k)] .
$$

Focusing on the derivatives of $g(x-1-k / b))$ at $x=k / b$, we get

$$
D^{m}[g(x-1-k / b)](k / b)=g^{(m)}(-1)=0
$$

for $m=0,1, \ldots, n$. Hence, as a consequence of the Leibniz rule (3.1),

$$
\gamma_{k}^{(m)}(k / b)=0, \quad \text { for } m=1,2, \ldots, n \text {. }
$$

The calculations for $x=-k / b, k=1, \ldots, k_{\max }$ are similar to the ones above hence we leave these to the reader.

$C^{n}$-smoothness at $x= \pm(k+1), k=1, \ldots, k_{\max }$. Again, we first consider $x=k+1$, $k=1, \ldots, k_{\max }$. For $x \in[k / b, k+1]$, we consider $\gamma_{k}(x)$ as a product of the two functions

$$
\prod_{j \in[k]} \frac{g(x-k-1-j(1 / b-1))}{g(x-k-j(1 / b-1))}
$$

and

$$
g(x-k-1) z(x-k)+b \psi(x-k) .
$$

We focus on the second of the two and observe that, for each $m=1,2, \ldots, n$,

$$
D^{m}[g(\cdot-k-1) z(\cdot-k)+b \psi(\cdot-k)](k+1)=\sum_{\ell=0}^{m}\left(\begin{array}{c}
m \\
\ell
\end{array}\right) g^{(\ell)}(0) z^{(m-\ell)}(1)+b \psi^{(m)}(0)
$$




$$
=g(0)\left[z^{(m)}(1)+\sum_{\ell=1}^{m}\left(\begin{array}{c}
m \\
\ell
\end{array}\right) \frac{g^{(\ell)}(0)}{g(0)} z^{(m-\ell)}(1)+b \frac{\psi^{(m)}(0)}{g(0)}\right]=0,
$$

where the final equality follows from the assumption (3.9). Thus, as a consequence of the Leibniz rule (3.1), we arrive at

$$
\gamma_{k}^{(m)}(k+1)=0
$$

for $m=1,2, \ldots, n$. The calculations for $x=-k-1, k=1, \ldots, k_{\max }$ are similar to the ones above, hence we leave these to the reader.

The proof of assertion (ii) $\Rightarrow(\mathrm{i})$ is similar to the argument in the proof of Lemma 3.6, hence we will omit the proof.

Example 3.2. We compute (3.8) and (3.9) for $n=2$ using Example 3.1. For $m=1$, using that $z$ also has to satisfy (3.5) and (3.6), we get

$$
z^{(1)}(0)=-z^{(1)}(1)=-2 b \frac{g^{(1)}(0)}{g(0)^{3}} .
$$

Similarly, for $m=2$, using that $z$ satisfies (3.5), (3.6), and (3.10), we get

$$
z^{(2)}(0)=-z^{(2)}(1)=6 b \frac{g^{(1)}(0)^{2}}{g(0)^{4}}-2 b \frac{g^{(2)}(0)}{g(0)^{3}} .
$$

It is easy to find a function $z:[0,1] \rightarrow \mathbb{C}$ satisfying the conditions in (i) in Theorem 3.8. E.g., if $n<\infty$, a polynomial $z$ of degree $2 n+2$ will always do. Further, as the conditions on $z \in C^{n}(\mathbb{R})$ only concern the derivatives of $z(x)$ at the boundary points $x=0$ and $x=1$, there is an abundance of $C^{n}$ dual windows of each $g \in V_{+}^{n}(\mathbb{R})$ for any value of $b \in(0,1)$. Hence, given a window $g \in V_{+}^{n}(\mathbb{R})$, we can easily construct dual windows in $C^{n}(\mathbb{R})$ using Theorem 3.8; example of such constructions will be given in Section 4.

We now exhibit a large class of window functions $g \in V_{+}^{n}(\mathbb{R})$ containing, e.g., all symmetric windows and all windows forming a partition of unity, for which the issue of computing $\psi^{(m)}(0)$ used in Theorem 3.8 disappears.

Corollary 3.9. Let $n \in \mathbb{Z}_{>0} \cup\{\infty\}$. Suppose $g \in V_{+}^{n}(\mathbb{R})$ satisfies $g^{(m)}(0)=0$ for $m=1, \ldots, n$. Then the following assertions are equivalent:

(i) $z \in C^{n}([0,1])$ satisfies (3.5), (3.6), and, for each $m=1, \ldots, n$,

$$
z^{(m)}(0)=0
$$

and

$$
z^{(m)}(1)=0
$$


(ii) $h_{z} \in C^{n}(\mathbb{R})$.

In particular, if

$$
z(x)=\frac{b}{g(0)^{3}}[2 g(x)-g(0)]
$$

then $h_{z} \in C^{n}(\mathbb{R})$.

Proof. By Lemma 3.7, it follows that $\psi^{(m)}(0)=0$ for $m=1, \ldots, n$. With $g^{(m)}(0)=0$ and $\psi^{(m)}(0)=0$ for all $m=1, \ldots, n$ conditions (3.8) and (3.9) reduce to (3.11) and (3.12), respectively. Finally, it is straightforward to verify that $z$ defined by (3.13) satisfies the $2 n+2$ conditions in (i).

Remark 3.10. (a) Suppose $g \in V_{+}^{n}(\mathbb{R})$ satisfies the assumptions of Corollary 3.9, i.e., $g^{(m)}(0)=0$ for $m=1, \ldots, n$. Now, if $h \in C^{n}(\mathbb{R})$ and $b \in \mathbb{C}$ satisfy the window condition $\sum_{n \in \mathbb{Z}} g(x+n) \overline{h(x+n)}=b$ for $x \in \mathbb{R}$, then by term-wise differentiating the window condition we see that $h^{(m)}(0)=0$ for $m=1, \ldots, n$. Thus, any dual window in $C^{n}(\mathbb{R})$, not necessarily with compact support, will also have this property.

(b) Suppose $g \in V_{+}^{n}(\mathbb{R})$ satisfies either $\sum_{n \in \mathbb{Z}} g(x+n)=1$ or $g(x)=g(-x)$ for $x \in \mathbb{R}$ (or both). Then $g$ satisfies the assumptions of Corollary 3.9, i.e., $g^{(m)}(0)=0$ for $m=1, \ldots, n$. For $g$ symmetric, this is obvious. If $g \in V_{+}^{n}(\mathbb{R})$ forms a partition of unity, then, by differentiating $g(x)+g(x-1)=1$ for $x \in[0,1]$, one will see that $g^{(m)}(0)=0$ for $m=1, \ldots, n$.

(c) The dual window $h_{z}$ defined by (3.13) in Corollary 3.9 is often a convenient choice as it guarantees that the dual window $h_{z}$ is defined only in terms of the window $g$. Hence, if $g$ is, e.g., a piecewise polynomial, then $h_{z}$ becomes a piecewise rational function of polynomials. However, if $g$ is symmetric, $h_{z}$ defined by (3.13) will only be symmetric, if $g(x)=g(0)-g(1-x)$ for $x \in[0,1]$, that is, if the graph of $g$, restricted to $[0,1] \times \mathbb{C}$, is anti-symmetric around $(1 / 2, g(0) / 2)$.

\subsection{Optimality of smoothness}

The first result of this section shows that, even though there are an abundance of dual windows in $C^{n}(\mathbb{R})$ for $g \in V_{+}^{n}(\mathbb{R}) \backslash C^{n+1}(\mathbb{R})$, additional smoothness, e.g., dual windows in $C^{n+1}(\mathbb{R})$, is in general not possible.

Proposition 3.11. Let $b \in(0,1)$. Let $n \in \mathbb{Z}_{\geq 0}$, and let $g \in V_{+}^{n}(\mathbb{R})$ with $\sum_{n \in \mathbb{Z}} g(x+n)=$ 1 for $x \in \mathbb{R}$. Assume $g$ is a real-valued, piecewise $C^{n+1}$-function for which $g^{(n+1)}$ has a simple discontinuity at $x=-1, x=0$, and/or $x=1$. If $h \in L^{2}(\mathbb{R})$ is compactly supported in $\left[-k_{\max }-1, k_{\max }+1\right]$, and $\left\{M_{b m} T_{k} g\right\}_{m, k \in \mathbb{Z}}$ and $\left\{M_{b m} T_{k} h\right\}_{m, k \in \mathbb{Z}}$ are dual frames, then $h \notin C^{n+1}(\mathbb{R})$.

Proof. Assume towards a contradiction that $h=h_{z} \in C^{n+1}(\mathbb{R})$. Recall that $g \in V_{+}^{n}(\mathbb{R})$ implies $g^{(m)}( \pm 1)=0$ for $m=0, \ldots, n$. We only consider the case $g(x)>0$ for $x \in$ $(-1,1)$ as the argument for $g(x)<0$ is similar. Since $g(x)>0$ for $x \in(-1,1)$, it 
follows that $g^{(n+1)}\left(-1^{+}\right) \geq 0$ and $g^{(n+1)}\left(1^{-}\right) \leq 0$. Assume that $g^{(n+1)}$ is discontinuous at $x=-1$ and/or $x=1$, i.e., $g^{(n+1)}\left(-1^{+}\right)>0$ and/or $g^{(n+1)}\left(1^{-}\right)<0$. The case $x=0$ follows from Theorem 3.13 below.

As in the proof of Theorem 3.8, we see that

$$
\overline{h_{z}^{(n+1)}\left(0^{-}\right)}=g^{n+1}\left(1^{-}\right) z(1)=-g^{n+1}\left(1^{-}\right) b / g(0)^{2}
$$

and

$$
\overline{h_{z}^{(n+1)}\left(0^{+}\right)}=-g^{n+1}\left(-1^{+}\right) z(0)=-g^{n+1}\left(-1^{+}\right) b / g(0)^{2}
$$

Since, by assumption, $h_{z}^{(n+1)}\left(0^{-}\right)=h_{z}^{(n+1)}\left(0^{+}\right)$, it follows that $g^{n+1}\left(1^{-}\right)=g^{n+1}\left(-1^{+}\right)$, which is a contradiction.

Example 3.3. Let $g(x)=\max (0,1-|x|)$ be the second cardinal B-spline with uniform knots. For any $b \in(0,1)$, the Gabor system $\left\{M_{b m} T_{k} g\right\}_{m, k \in \mathbb{Z}}$ is a frame for $L^{2}(\mathbb{R})$. Since $g^{\prime}(x)$ is discontinuous at $x=-1$ (and at $x=0$ and $x=1$ ), it follows from Proposition 3.11 that no dual windows $h \in C^{1}(\mathbb{R}) \cap L^{2}(\mathbb{R})$ with support in $\left[-k_{\max }-1, k_{\max }+1\right]$ exists for any value of $b$.

Let us comment on the assumptions of Proposition 3.11. The location of the discontinuity of $g^{(n+1)}$ is important. In fact, if discontinuities of $g^{(n+1)}$ avoid certain points, the conclusion $h \notin C^{n+1}(\mathbb{R})$ of Proposition 3.11 may not hold [21]. On the other hand, we assume the partition of unity property of $g$ only for convenience as to simplify the proof. Furthermore, as we see by the next two results, positivity of $|g(x)|$ on $(-1,1)$ and compact support of the dual window $h$ are also not essential for obstructions results on the smoothness of dual windows. For $n=-1$, we ignore the requirement $g \in C^{n}(\mathbb{R})$ in the formulation below.

Lemma 3.12. Let $n \in \mathbb{Z}_{\geq-1}$, and let $g \in C^{n}(\mathbb{R})$ be a piecewise $C^{n+1}$-function. Let $\left\{x_{r}\right\}_{r \in[R]}=\left\{x_{1}, \ldots, x_{R}\right\}$ denote the finite set of points, where $g^{(n+1)}$ has simple discontinuities. Assume $h \in C^{n+1}(\mathbb{R})$ and the constants $a>0, b \in \mathbb{C}$ satisfy the window condition

$$
\sum_{n \in \mathbb{Z}} g(x+a n) \overline{h(x+a n)}=b, \quad \text { for all } x \in[-a / 2, a / 2] .
$$

Let $\left\{t_{r}\right\}_{r \in[R]}=\left\{x_{r}\right\}_{r \in[R]}(\bmod a)$ so that $t_{r} \in[-a / 2, a / 2)$ and $t_{r} \leq t_{r+1}$. Set $t_{0}=$ $-a / 2$ and $t_{R+1}=a / 2$. Suppose $\sum_{n \in \mathbb{Z}} D^{m}[g(\cdot+a n) \overline{h(\cdot+a n)}]$ converges uniformly on $\left[t_{r}, t_{r+1}\right]$ for $m=1, \ldots, n+1$ and $r=0, \ldots, R$. Then, for each $r=1, \ldots, R$,

$$
\sum_{\left\{s \in[R]: x_{s}-x_{r} \in a \mathbb{Z}\right\}}\left[g^{(n+1)}\left(x_{s}^{+}\right)-g^{(n+1)}\left(x_{s}^{-}\right)\right] h\left(x_{r}\right)=0 .
$$

In particular, if $\left\{s \in[R]: x_{s}-x_{r} \in a \mathbb{Z}\right\}=\{r\}$, then $h\left(x_{r}\right)=0$.

Proof. The following argument is inspired by the proof of Lemma 1 in [19]. Fix $r \in$ $[R]$. Uniform convergence of $\sum_{n \in \mathbb{Z}} D^{m}[g(\cdot+a n) \overline{h(\cdot+a n)}]$ for each $m=0,1, \ldots, n+1$ 
allows us to differentiate the window condition (3.14) term by term [27, Theorem 7.17]. Differentiating $m$ times then gives:

$$
\sum_{n \in \mathbb{Z}} \sum_{\ell=0}^{m}\left(\begin{array}{c}
m \\
\ell
\end{array}\right) g^{(\ell)}(x+a n) \overline{h^{(m-\ell)}(x+a n)}=0, \quad \text { for all } x \in\left(t_{r}, t_{r+1}\right) .
$$

for all $r=0,1, \ldots, R$ and $m=1, \ldots, n$. Note that the sum (3.16) is $a$ periodic. Hence, by subtracting the two one-sided limits $x \nearrow t_{r}$ and $x \searrow t_{r}$ of (3.16) with $m=n+1$, we obtain (3.15).

Recall that if $\left\{M_{b m} T_{a k} g\right\}_{m, k \in \mathbb{Z}}$ and $\left\{M_{b m} T_{a k} h\right\}_{m, k \in \mathbb{Z}}$ with $g, h \in L^{2}(\mathbb{R})$ are dual frames for $L^{2}(\mathbb{R})$, then (3.14) holds. Hence, under the assumptions of Lemma 3.12, duality of $g$ and $h$ restricts the possible values of $h$ on $\left\{x_{j}\right\}_{j \in J}$. For windows $g$ with support in $[-1,1]$, Lemma 3.12 leads to the following general obstruction result.

Theorem 3.13. Let $b \in(0,1)$ and $h \in L^{2}(\mathbb{R})$. Let $n \in \mathbb{Z}_{\geq-1}$, and let $g \in C^{n}(\mathbb{R})$ be a piecewise $C^{n+1}$-function with $\operatorname{supp} g \subset[-1,1]$, and let $\left\{x_{j}\right\}_{j \in J} \subset[-1,1]$ denote the finite set of points, where $g^{(n+1)}$ is discontinuous. Assume either

(i) $0 \in\left\{x_{j}\right\}_{j \in J}$ and $\operatorname{supp} h \subset\left[-k_{\max }-1, k_{\max }+1\right]$, or

(ii) $0 \in\left\{x_{j}\right\}_{j \in J}$ and $\pm 1 \notin\left\{x_{j}\right\}_{j \in J}$.

If $\left\{M_{b m} T_{k} g\right\}_{m, k \in \mathbb{Z}}$ and $\left\{M_{b m} T_{k} h\right\}_{m, k \in \mathbb{Z}}$ are dual frames, then $h \notin C^{n+1}(\mathbb{R})$.

Proof. Assume towards a contradiction that $h \in C^{n+1}(\mathbb{R})$. From $\operatorname{supp} g \subset[-1,1]$, it follows that $g(k)=0$ for all $k \in \mathbb{Z} \backslash\{0\}$ and that $\left\{x_{j}\right\}_{j \in J} \subset[-1,1]$. Hence, equation (3.2) for $k=0$ implies that $h(0)=b / g(0)>0$. Depending on whether we use assumption (i) or (ii), the points $x= \pm 1$ may or may not belong to $\left\{x_{j}\right\}_{j \in J}$. In either case, $g^{(n+1)}\left(-1^{-}\right)=g^{(n+1)}\left(1^{+}\right)=0$ and we have from (3.15) that

$\left.0=\left[g^{(n+1)}\left(-1^{+}\right)-0\right)\right] h(-1)+\left[g^{(n+1)}\left(0^{+}\right)-g^{(n+1)}\left(0^{-}\right)\right] h(0)+\left[0-g^{(n+1)}\left(1^{-}\right)\right] h(1)$

If we use assumption (i), then, by the compact support of $h$, we have from [6, Lemma 3.2] that $h( \pm 1)=0$. On the other hand, from assumption (ii), we have $g^{(n+1)}\left(-1^{+}\right)=$ $g^{(n+1)}\left(1^{-}\right)=0$. In either case, we get

$$
\left[g^{(n+1)}\left(0^{+}\right)-g^{(n+1)}\left(0^{-}\right)\right] h(0)=0,
$$

which is a contradiction to $0 \in\left\{x_{j}\right\}_{j \in J}$ and $h(0)>0$.

The conditions on the window $g$ in the above results should be understood as follows. In order to make the statement as strong as possible, we want generators $g$ just shy of being in the $C^{n+1}$-class. Hence, the function $g$ is assumed to be $C^{n}$ everywhere and piecewise $C^{n+1}$ except at a finite number of points, where both one-sided limits of $g^{(n+1)}$ exist, but do not agree. The following example shows a general, but typical, obstruction of the smoothness of dual windows. 
Example 3.4. Let $b \in(0,1), n \in \mathbb{Z}_{>0}$, and let $g \in C^{n}(\mathbb{R})$ with $\operatorname{supp} g \subset[-1,1]$ be a $C^{\infty}$-function except at $x=0$, where $g^{(n+1)}$ fails to be continuous. Suppose the Gabor system $\left\{M_{b m} T_{k} g\right\}_{m, k \in \mathbb{Z}}$ is a frame for $L^{2}(\mathbb{R})$. Then, by Theorem 3.13, it follows that no dual windows $h \in C^{n+1}(\mathbb{R}) \cap L^{2}(\mathbb{R})$ exists. Note that this conclusion holds whether or not $h$ is assumed to have compact support.

\subsection{Small support}

While the previous section considered optimality of the smoothness of the dual windows, we are here concerned with optimizing, that is, minimizing, the support length. Such questions were considered in [7], where the authors characterized the existence of continuous dual windows with short support for continuous windows $g$ with finitely many zeros inside their support $[-1,1]$. In the following result we consider the possibility of higher order smoothness of dual windows with short support.

Theorem 3.14. Let $n \in \mathbb{Z}_{\geq 0}$, let $b \in\left[\frac{N}{N+1}, \frac{2 N}{2 N+1}\right)$ for some $N \in \mathbb{Z}_{>0}$, and let $g \in$ $V_{+}^{n}(\mathbb{R})$. Define

$$
z(x)= \begin{cases}b \frac{\psi(x)}{g(x)} & x \in\left[0,1-N\left(\frac{1}{b}-1\right)\right] \\ z_{\text {mid }}(x) & x \in\left(1-N\left(\frac{1}{b}-1\right), N\left(\frac{1}{b}-1\right)\right) \\ -b \frac{\psi(x)}{g(x-1)} & x \in\left[N\left(\frac{1}{b}-1\right), 1\right]\end{cases}
$$

where $z_{\text {mid }}:\left(1-N\left(\frac{1}{b}-1\right), N\left(\frac{1}{b}-1\right)\right) \rightarrow \mathbb{C}$ is a measurable function. The following assertions hold:

(a) The dual window $h_{z}$ has compact support in $[-N, N]$.

(b) $h_{z} \in C^{n}(\mathbb{R})$ if and only if $z \in C^{n}([0,1])$.

(c) Suppose $g$ is even. Then $h_{z}$ is even if and only if $z_{\text {mid }}$ is antisymmetric around $x=1 / 2$, i.e., $z_{\text {mid }}(x)=-z_{\text {mid }}(1-x)$ for a.e. $x \in\left(1-N\left(\frac{1}{b}-1\right), 1 / 2\right]$.

Proof. By definition of $N$, we have $N\left(\frac{1}{b}-1\right) \leq 1$, hence, $z$ is well-defined.

(a): If $k_{\max }<N$, then $\operatorname{supp} h_{z} \subset\left[-k_{\max }-1, k_{\max }+1\right] \subset[-N, N]$ by (2.3). Assume now that $k_{\max } \geq N$ and consider the dual windows $h_{z}$ on $\left[N, k_{\max }+1\right]$. For $x>N$, it suffices to show that $h_{z}(x)=0$ for $x \in(k / b, k+1)$ and $k=N, \ldots, k_{\max }$. Recall that, for any $k=1, \ldots, k_{\max }$,

$$
\overline{h_{z}(x)}=\gamma_{k}(x)
$$

for $x \in(k / b, k+1)$, which can be rewritten as

$$
\overline{h_{z}(x+k)}=\gamma_{k}(x+k)=(-1)^{k} \prod_{j \in[k]} \frac{g(x-1-j(1 / b-1))}{g(x-j(1 / b-1))}[g(x-1) z(x)+b \psi(x)]
$$

for $x \in(k / b-k, 1)$. Since $k \geq N$, we have the inclusion $\left(k\left(\frac{1}{b}-1\right), 1\right) \subset\left(N\left(\frac{1}{b}-1\right), 1\right)$. Hence

$$
g(x-1) z(x)+b \psi(x)=g(x-1)\left(-b \frac{\psi(x)}{g(x-1)}\right)+b \psi(x)=0 .
$$


Thus $h_{z}(x)=0$ for $x>N$. The argument for $x<-N$ is similar so we omit it.

(b): By inserting $x=0$ and $x=1$ into (3.17), it can easily be seen that $z$ satisfies (3.5) and (3.6), respectively. Therefore, the result for $n=0$ simply follows from Lemma 3.6. For $n>0$, the "only if"-assertion follows directly from Theorem 3.8. To prove the other direction, we assume that $z \in C^{n}([0,1])$. From definition (3.17) we have:

$$
g(x) z(x)-b \psi(x)=0 \quad \text { for all } x \in\left[0,1-N\left(\frac{1}{b}-1\right)\right] .
$$

By differentiating both sides $m$ times, isolating $z^{(m)}(x)$ and inserting $x=0$, we see that $z$ satisfies (3.8). In a similar way, one proves that $z$ satisfies (3.9). Hence, by Theorem 3.8, $h_{z} \in C^{n}(\mathbb{R})$.

(c): From (3.17) we see that, for $x \in\left[0,1-N\left(\frac{1}{b}-1\right)\right]$, the function $z$ satisfies

$$
-z(1-x)=b \frac{\psi(1-x)}{g(-x)}=b \frac{\psi(x)}{g(x)}=z(x),
$$

where the second equality uses that $g$ and $\psi$ are even and that $\psi$ is 1 -periodic. Hence, if $g$ is even, then $z_{m i d}$ is antisymmetric around $x=1 / 2$ if and only if $z$ defined by (3.17) is antisymmetric around $x=1 / 2$. The conclusion now follows from Lemma 3.5.

The short support of Theorem 3.14 is optimal in the following sense of [7]: If a dual window $h$ with support supp $h \subset[-N, N]$ exists, then necessarily $b \leq 2 N /(2 N+1)$, see [7, Theorem 2.3]. If, in addition, $h$ is assumed to be continuous, then $b<2 N /(2 N+1)$, see [7, Theorem 2.5].

\section{Examples of the construction}

In this section, we present two examples of the construction procedure of dual windows using the results from the previous sections. In Example 4.1 we construct dual windows of the classical and widely used Hann and Blackman window, respectively. In Example 4.2 we consider a smoother, but non-symmetric window; the setup is more complicated than Example 4.1 and perhaps less useful for applications, but it serves as a proof of concept of the flexibility of our method.

Example 4.1. The Hann window $g_{\text {hann }} \in C^{1}(\mathbb{R})$ is defined by

$$
g_{\text {hann }}(x)=\cos ^{2}(\pi x / 2) \mathbf{1}_{[-1,1]}(x)= \begin{cases}\frac{1}{2}-\frac{1}{2} \cos (\pi(x+1)) & x \in[-1,0) \\ \frac{1}{2}+\frac{1}{2} \cos (\pi(x)) & x \in[0,1] \\ 0 & \text { otherwise }\end{cases}
$$

and the Blackman window $g_{\text {blac }} \in C^{1}(\mathbb{R})$ is defined by

$$
g_{\text {blac }}(x)=[0.42+0.5 \cos (\pi x)+0.08 \cos (2 \pi x)] \mathbf{1}_{[-1,1]}(x)
$$

for $x \in \mathbb{R}$, see Figure 2. Both these widows are continuously differentiable, symmetric, non-negative, and normalized $g(0)=1$, and the Hann window even has the partition 

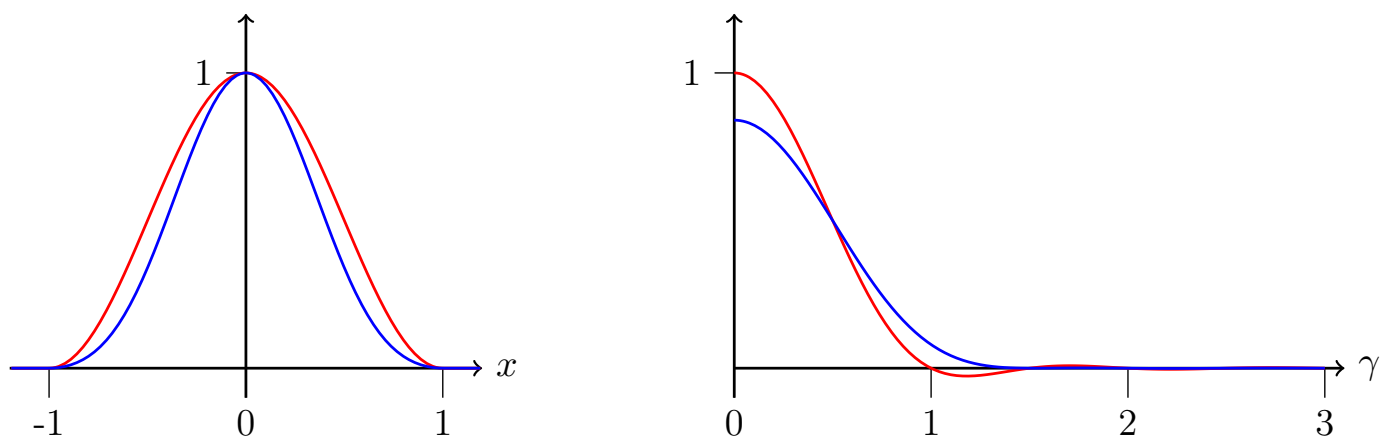

Figure 2: Left: the Hann window $g_{\text {hann }} \in V_{+}^{1}(\mathbb{R})\left(\right.$ red) and the Blackman window $g_{\text {blac }} \in V_{+}^{1}(\mathbb{R})$ (blue). Right: The Fourier transform of the Hann window $\widehat{g}_{\text {hann }}$ (red) and of the Blackman window $\widehat{g}_{\text {blac }}$ (blue). Both windows and their Fourier transforms are real and symmetric.

of unity property. Both of the windows belong to $V_{+}^{1}(\mathbb{R})$, but not $V_{+}^{2}(\mathbb{R})$; hence, the optimal smoothness of compactly supported dual windows are $h \in C^{1}(\mathbb{R})$.

As an example, let us consider the modulation parameter $b=3 / 5$. By definition of $k_{\max }$ we get $k_{\max }=1$. Thus, the dual windows $h_{z}$ defined in (2.2) will have support in $[-2,2]$.

Since $g$ is a trigonometric polynomial on $[-1,1]$, it is natural to take $z$ to be a trigonometric polynomial as well. For the Hann window the standard choice (3.13) is:

$$
z_{\text {hann }}(x)=b \cos (\pi x) \quad \text { for } x \in[0,1],
$$

while (3.13) for the Blackman window becomes:

$$
z_{\text {blac }}(x)=b[-0.16+0.5 \cos (\pi x)+0.08 \cos (2 \pi x)] \quad \text { for } x \in[0,1] .
$$

Figure 3 shows dual windows of the Hann window $h_{\text {hann }}$ and of the Blackman window $h_{\text {blac }}$ defined using $z$ from (4.1) and (4.2), respectively. While $z_{\text {hann }}$ is anti-symmetric around $x=1 / 2$, this is not the case for the chosen $z_{\text {blac }}$; see Lemma 3.5 and Remark 3.10(c).

We can actually decrease the support size of the dual windows without sacrificing the $C^{1}$-smoothness. By applying Theorem 3.14 with $N=1$ and taking $z_{\text {mid }}$ to be the unique third degree trigonometric polynomial $z_{m i d}(x)=c_{1} \cos (\pi x)+c_{3} \cos (3 \pi x)$ so that $z \in C^{1}(\mathbb{R})$, we obtain dual windows in $C^{1}(\mathbb{R})$ with support in $[-1,1]$. It turns out that the support of the dual windows even shrink to $[-2 / 3,2 / 3]$ for this specific setup. Since the two constructed functions $z_{m i d}$ are anti-symmetric around $x=1 / 2$, it follows by Theorem 3.14(c) that both these dual windows will be symmetric. The short-support dual windows of the Hann and Blackman window and their Fourier transforms are shown in Figure 4.

The next example illustrates the construction of dual windows when $g$ does not have zero derivatives at the origin and the redundancy $(a b)^{-1}=3 \pi / 7$ is irrational. 


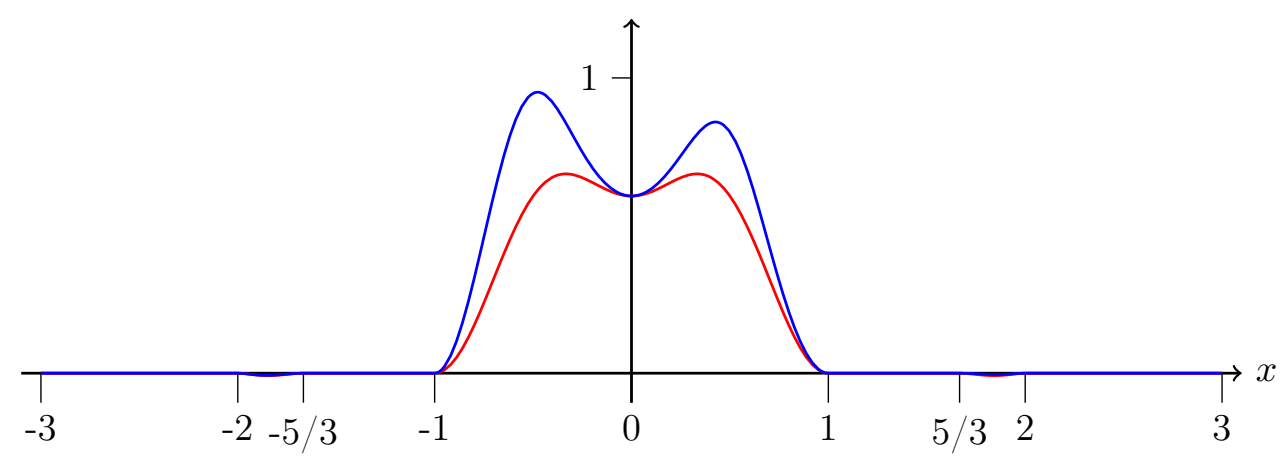

Figure 3: Dual windows $h_{\text {hann }}$ (red) and $h_{\text {blac }}$ (blue) of the Hann and Blackman window based on $z_{\text {hann }}$ and $z_{\text {blac }}$ defined in $(4.1)$ and $(4.2)$, respectively. Both windows are in $C^{1}(\mathbb{R})$ and with support $\operatorname{supp} h=[-2,-5 / 3] \cup[-1,1] \cup[5 / 3,2]$.
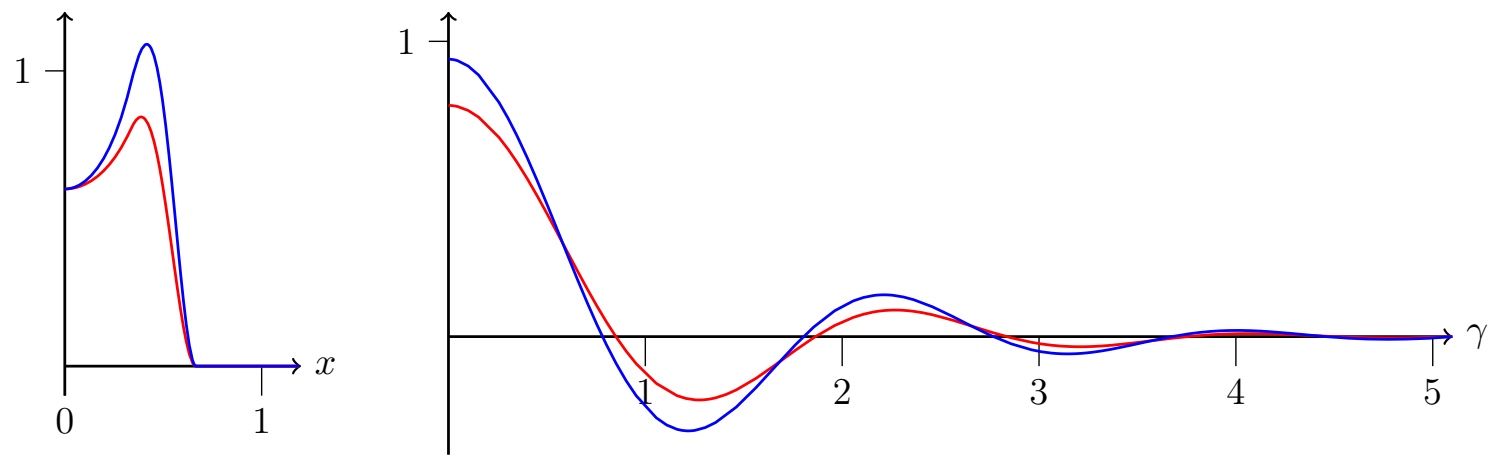

Figure 4: Left: Dual windows in $C^{1}(\mathbb{R})$ with short support on $[-2 / 3,2 / 3]$. The dual of the Hann window is shown in red, while the dual of the Blackman window is shown in blue. Right: The Fourier transforms of the two dual windows shown left: the Fourier transform of the dual Hann window (red) and the Fourier transform of the dual Blackman window (blue). Both dual windows and their Fourier transforms are real and symmetric.

Example 4.2. We take $\beta$ to be a spline defined as:

$$
\beta(x)= \begin{cases}p(x) & x \in[-1,-4 / 5] \\ 1 & x \in[-4 / 5,4 / 5] \\ p(-x) & x \in[4 / 5,1] \\ 0 & \text { otherwise }\end{cases}
$$

where $p(x)=10625-60000 x+135000 x^{2}-151250 x^{3}+84375 x^{4}-18750 x^{5}$ is the five-degree polynomial satisfying $p(1)=p^{\prime}(1)=p^{\prime \prime}(1)=p^{\prime}(4 / 5)=p^{\prime \prime}(4 / 5)=0$ and $p(4 / 5)=1$. Then $\beta \in C^{2}(\mathbb{R})$ is a bump function supported on $[-1,1]$. We consider the window $g \in V_{+}^{2}(\mathbb{R})$ defined by

$$
g(x)=\frac{1}{16}(2-(x-5)(x+3)) \beta(x)
$$




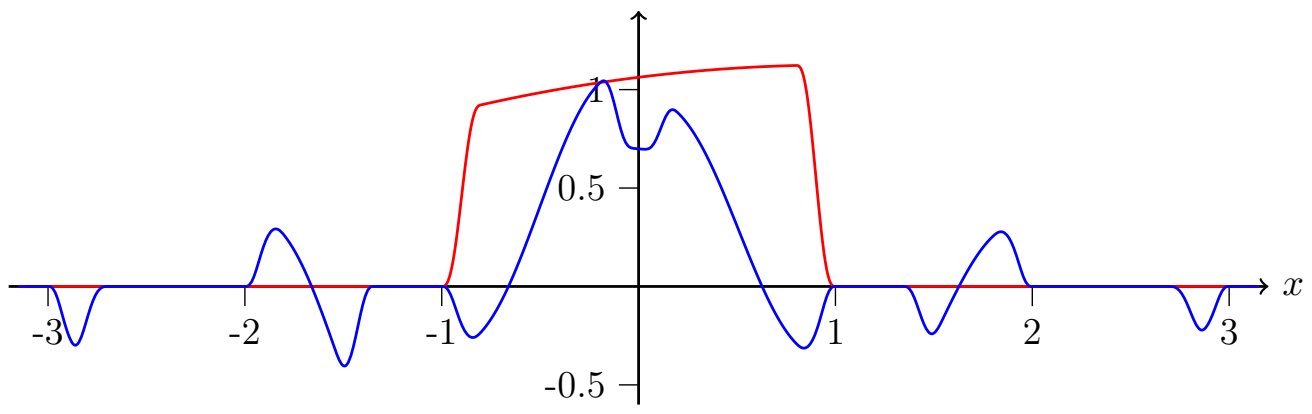

Figure 5: The window function $g \in V_{+}^{2}(\mathbb{R})$ (red) and a dual window $h_{z}$ (blue) for $b=\frac{7}{3 \pi}$. Both $g$ and $h$ are $C^{2}$-functions, and $h$ has support in $[-3,3]$.

As an example of an irrational modulation parameter, let us consider $b=\frac{7}{3 \pi}$. Then $k_{\max }=2$ so the support of $h_{z}$ is:

$$
\operatorname{supp} h_{z}=[-3,-6 \pi / 7] \cup[-2,-3 \pi / 7] \cup[-1,1] \cup[3 \pi / 7,2] \cup[6 \pi / 7,3] \subset[-3,3] .
$$

We chose $z$ to be the unique polynomial of degree five that satisfies the six conditions of Theorem 3.8 for $n=2$; these conditions are explicitly computed in Example 3.2. It follows that $h_{z} \in C^{2}(\mathbb{R})$. The graphs of $g$ and the dual window $h_{z}$ are shown in Figure 5 .

\section{References}

[1] A. G. D. Atindehou, Y. B. Kouagou, and K. A. Okoudjou. Frame sets for a class of compactly supported continuous functions. Asian-Eur. J. Math., to appear in print. doi:10.1142/S179355712050093X.

[2] P. Balazs, H. G. Feichtinger, M. Hampejs, and G. Kracher. Double preconditioning for gabor frames. IEEE Transactions on Signal Processing, 54(12):4597-4610, dec 2006. doi:10.1109/tsp.2006.882100.

[3] O. Christensen. An introduction to frames and Riesz bases. Applied and Numerical Harmonic Analysis. Birkhäuser/Springer, [Cham], second edition, 2016. doi:10 . 1007/978-3-319-25613-9.

[4] O. Christensen. Pairs of dual Gabor frame generators with compact support and desired frequency localization. Appl. Comput. Harmon. Anal., 20(3):403-410, 2006. doi:10.1016/j.acha.2005.10.003.

[5] O. Christensen and S. S. Goh. From dual pairs of Gabor frames to dual pairs of wavelet frames and vice versa. Appl. Comput. Harmon. Anal., 36(2):198-214, 2014. doi:10.1016/j.acha.2013.03.005.

[6] O. Christensen, H. O. Kim, and R. Y. Kim. Gabor windows supported on $[-1,1]$ and compactly supported dual windows. Appl. Comput. Harmon. Anal., 28(1):89103, 2010. doi:10.1016/j.acha.2009.07.004. 
[7] O. Christensen, H. O. Kim, and R. Y. Kim. Gabor windows supported on $[-1,1]$ and dual windows with small support. Adv. Comput. Math., 36(4):525-545, 2012. doi:10.1007/s10444-011-9189-0.

[8] O. Christensen, H. O. Kim, and R. Y. Kim. On Gabor frames generated by signchanging windows and B-splines. Appl. Comput. Harmon. Anal., 39(3):534-544, 2015. doi:10.1016/j.acha.2015.02.006.

[9] O. Christensen, H. O. Kim, and R. Y. Kim. On the Gabor frame set for compactly supported continuous functions. J. Inequal. Appl., pages Paper No. 94, 17, 2016. doi:10.1186/s13660-016-1021-4.

[10] O. Christensen and R. Y. Kim. Pairs of explicitly given dual Gabor frames in $L^{2}\left(\mathbb{R}^{d}\right)$. J. Fourier Anal. Appl., 12(3):243-255, 2006. doi:10.1007/ s00041-005-5052-3.

[11] I. Daubechies, A. Grossmann, and Y. Meyer. Painless nonorthogonal expansions. J. Math. Phys., 27(5):1271-1283, 1986. doi:10.1063/1.527388.

[12] V. Del Prete. Estimates, decay properties, and computation of the dual function for Gabor frames. J. Fourier Anal. Appl., 5(6):545-562, 1999. doi:10.1007/ BF01257190.

[13] K. Gröchenig and J. Stöckler. Gabor frames and totally positive functions. Duke Math. J., 162(6):1003-1031, 2013. doi:10.1215/00127094-2141944.

[14] A. J. E. M. Janssen. The duality condition for Weyl-Heisenberg frames. In Gabor analysis and algorithms, Appl. Numer. Harmon. Anal., pages 33-84. Birkhäuser Boston, Boston, MA, 1998.

[15] A. J. E. M. Janssen and P. L. Søndergaard. Iterative algorithms to approximate canonical Gabor windows: computational aspects. J. Fourier Anal. Appl., 13(2):211-241, 2007. doi:10.1007/s00041-006-6069-y.

[16] I. Kim. Gabor frames with trigonometric spline dual windows. Asian-Eur. J. Math., 8(4):1550072, 32, 2015. doi:10.1142/S1793557115500722.

[17] T. Kloos and J. Stöckler. Zak transforms and Gabor frames of totally positive functions and exponential B-splines. J. Approx. Theory, 184:209-237, 2014. doi: $10.1016 / j \cdot j$ at. 2014.05.010.

[18] T. Kloos, J. Stöckler, and K. Gröchenig. Implementation of discretized Gabor frames and their duals. IEEE Trans. Inform. Theory, 62(5):2759-2771, 2016. doi: 10.1109/TIT.2016.2541918.

[19] R. S. Laugesen. Gabor dual spline windows. Appl. Comput. Harmon. Anal., 27(2):180-194, 2009. doi:10.1016/j .acha.2009.02.002. 
[20] J. Lemvig and K. H. Nielsen. Counterexamples to the B-spline conjecture for Gabor frames. J. Fourier Anal. Appl., 22(6):1440-1451, 2016. doi:10.1007/ s00041-016-9462-1.

[21] J. Lemvig and K. H. Nielsen. On the smoothness of dual windows for gabor windows supported on $[-1,1]$. In 2019 International Conference on Sampling Theory and Applications (SampTA), IEEE Xplore, 2019.

[22] S. Li. On general frame decompositions. Numer. Funct. Anal. Optim., 16(910):1181-1191, 1995. doi:10.1080/01630569508816668.

[23] S. Moreno-Picot, F. J. Ferri, M. Arevalillo-Herraez, and W. Diaz-Villanueva. Efficient analysis and synthesis using a new factorization of the gabor frame matrix. IEEE Transactions on Signal Processing, 66(17):4564-4573, sep 2018. doi: 10.1109/tsp.2018.2855643.

[24] N. Perraudin, N. Holighaus, P. L. Sø ndergaard, and P. Balazs. Designing Gabor windows using convex optimization. Appl. Math. Comput., 330:266-287, 2018. doi: $10.1016 / j$. amc. 2018.01.035.

[25] A. Ron and Z. Shen. Frames and stable bases for shift-invariant subspaces of $L_{2}\left(\mathbf{R}^{d}\right)$. Canad. J. Math., 47(5):1051-1094, 1995. doi:10.4153/CJM-1995-056-1.

[26] A. Ron and Z. Shen. Weyl-Heisenberg frames and Riesz bases in $L_{2}\left(\mathbf{R}^{d}\right)$. Duke Math. J., 89(2):237-282, 1997. doi:10.1215/S0012-7094-97-08913-4.

[27] W. Rudin. Principles of mathematical analysis. McGraw-Hill Book Co., New York, third edition, 1976. International Series in Pure and Applied Mathematics. 